\title{
Nature of the magnetic stripes in fully oxygenated La2CuO4+y
}

uueanu, Ana Elena; Jacobsen, Henrik; Jensen Ray, Pia; Holm-Dahlin, Sonja; Lctuu, Monica Elisabeta; Tejsner, Tim Birger; Grivel, Jean Claude; Schmidt, Wolfgang; Toft-Petersen, Rasmus; Steffens, Paul Total number of authors:

15

Published in:

Physical Review B

Link to article, DOI:

10.1103/PhysRevB.103.045138

Publication date:

2021

Document Version

Publisher's PDF, also known as Version of record

Link back to DTU Orbit

Citation (APA):

uueanu, A. E., Jacobsen, H., Jensen Ray, P., Holm-Dahlin, S., Lctuu, M. E., Tejsner, T. B., Grivel, J. C., Schmidt, W., Toft-Petersen, R., Steffens, P., Boehm, M., Wells, B., Udby, L., Lefmann, K., \& Rømer, A.'T. (2021). Nature of the magnetic stripes in fully oxygenated $\mathrm{La}_{2} \mathrm{CuO}_{4+\mathrm{y}}$. Physical Review B, 103(4), [045138].
https://doi.org/10.1103/PhysRevB.103.045138

\section{General rights}

Copyright and moral rights for the publications made accessible in the public portal are retained by the authors and/or other copyright owners and it is a condition of accessing publications that users recognise and abide by the legal requirements associated with these rights.

- Users may download and print one copy of any publication from the public portal for the purpose of private study or research.

- You may not further distribute the material or use it for any profit-making activity or commercial gain

- You may freely distribute the URL identifying the publication in the public portal 


\title{
Nature of the magnetic stripes in fully oxygenated $\mathrm{La}_{2} \mathrm{CuO}_{4+y}$
}

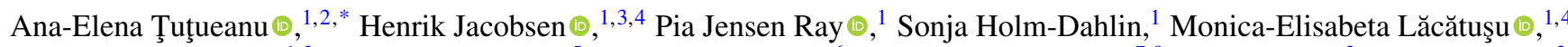 \\ Tim Birger Tejsner ${ }^{1,2}$ Jean-Claude Grivel, ${ }^{5}$ Wolfgang Schmidt, ${ }^{6}$ Rasmus Toft-Petersen, ${ }^{7,8}$ Paul Steffens, ${ }^{2}$ Martin Boehm, ${ }^{2}$ \\ Barrett Wells, ${ }^{9}$ Linda Udby, ${ }^{1}$ Kim Lefmann $\odot,{ }^{1}$ and Astrid Tranum Rømer ${ }^{1}$ \\ ${ }^{1}$ Nanoscience Center, Niels Bohr Institute, University of Copenhagen, 2100 Copenhagen, Denmark \\ ${ }^{2}$ Institut Laue-Langevin, 38042 Grenoble, France \\ ${ }^{3}$ Department of Physics, University of Oxford, OX1 3PU, United Kingdom \\ ${ }^{4}$ Paul Scherrer Institute, Laboratory for Neutron Scattering and Imaging, 5232 Villigen, Switzerland \\ ${ }^{5}$ Department of Energy Conversion, Technical University of Denmark, 4000 Roskilde, Denmark \\ ${ }^{6}$ Forschungszentrum Jülich GmbH, Jülich Centre for Neutron Science at ILL, 38042 Grenoble, France \\ ${ }^{7}$ Helmholtz Center Berlin for Materials and Energy, 14109 Berlin, Germany \\ ${ }^{8}$ Department of Physics, Technical University of Denmark, 2800 Kgs. Lyngby, Denmark \\ ${ }^{9}$ Department of Physics and Institute of Materials Science, University of Connecticut, Storrs, Connecticut 06269, USA
}

(Received 26 August 2020; revised 16 November 2020; accepted 5 January 2021; published 28 January 2021)

\begin{abstract}
We present triple-axis neutron scattering studies of static and dynamic magnetic stripes in an optimally oxygen-doped cuprate superconductor, $\mathrm{La}_{2} \mathrm{CuO}_{4+y}$, which exhibits a clean superconducting transition at $T_{c}=$ $42 \mathrm{~K}$. Polarization analysis reveals that the magnetic stripe structure is equally represented along both of the tetragonal crystal axes and that the fluctuating stripes display significant weight for in-plane as well as out-of-plane spin components. Both static magnetic order as well as low-energy fluctuations are fully developed in zero applied magnetic field and the low-energy spin fluctuations at $\hbar \omega=0.3-10 \mathrm{meV}$ intensify on cooling. We interpret this as an indication that superconductivity and low-energy spin fluctuations coexist microscopically in spatial regions which are separated from domains with static magnetic order.
\end{abstract}

DOI: 10.1103/PhysRevB.103.045138

\section{INTRODUCTION}

The interplay between magnetism and unconventional superconductivity (SC) remains controversial, inspiring much theoretical and experimental work in this field [1-5]. On the experimental side, one of the main pursuits is to establish key similarities in material properties across a range of existing superconductors, most of which differ in the details. For example, one such property is the magnetism, the fluctuations of which are thought to have an important role in the electron pairing mechanism [6]. Therefore, the magnetism of all known cuprate superconductors has been highly researched $[2,7,8]$ since their discovery, but a general consensus on its role in the pairing mechanism is still to be reached.

In this paper we address $\mathrm{La}_{2} \mathrm{CuO}_{4+y}$ (LCOO) which belongs to the family of single-layer cuprate superconductors $\mathrm{La}_{2-x} \mathrm{M}_{x} \mathrm{CuO}_{4+y}$, where the metal dopant $\mathrm{M}$ is either $\mathrm{Ba}$ (LBCO) or Sr (LSCO). In LCOO, however, the doping is provided solely by the excess oxygen ions. All La-based cuprates exhibit optimal superconductivity at doping values of around 0.15 holes per formula unit, producing quite similar superconducting transition temperatures $\left(T_{c}\right)$, in the range 32-42 K [9-11]. In addition, these systems display incommensurate magnetic order observed by neutron scattering at a quartet of incommensurate (IC) values of the scattering

\footnotetext{
*tutueanu@ill.fr
}

vector (in orthorhombic notation:) $\mathbf{Q}_{\mathrm{IC}}=\left(1 \pm \delta_{h}, \pm \delta_{k}, 0\right)$, where often $\delta_{h} \simeq \delta_{k} \simeq \delta=1 / 8$ [12-14]. The commensurate structure $(\delta=1 / 8)$ is consistent with a picture of antiferromagnetic (AFM) "stripes" of period 8 , as first observed in Nd-containing LSCO, $\mathrm{La}_{1.48} \mathrm{Nd}_{0.4} \mathrm{Sr}_{0.12} \mathrm{CuO}_{4}$, by Tranquada et al. [15] and later in LBCO [16,17], LCOO [13], and LSCO [18].

The magnetic order, also referred to as static stripes or spin-density-wave order (SDW), is generally regarded as a distinct electronic phase competing with uniform $d$-wave superconductivity, since it is predominantly present in underdoped samples with reduced $T_{c}$. This interpretation is supported by the fact that the magnetic ordering is more robust and survives to higher temperatures $\left(T_{N}\right)$ around the anomalous $x=1 / 8$ doping, where $T_{c}$ is significantly suppressed $[7,14,18,19]$. Additional support for the competition scenario is the observation of enhancement of the elastic IC response on application of an external magnetic field perpendicular to the $\mathrm{CuO}_{2}$ planes, indicating that suppression of the superconducting order goes hand-in-hand with a strengthening of the magnetic order [17,20-26]. The other way around, it was recently demonstrated that suppression of magnetic order by strain leads to a dramatic increase in $T_{c}$ in LBCO [27].

The corresponding magnetic fluctuations, also known as dynamic stripes, have been observed and comprehensively studied, especially in LSCO samples, over a wide range of dopings. In the absence of magnetic order, optimally doped LSCO samples exhibit gapped magnetic excitations with a 
gap of $\Delta \sim 6 \mathrm{meV}$ in the SC phase [28,29]. In contrast, for slightly lower dopings $x<0.13\left(T_{c} \leqslant 30 \mathrm{~K}\right)$, where static stripes are prominent, the presence of the superconducting phase is marked by the opening of an incomplete spin gap [11,30-32]. In both the optimally and underdoped cases, an applied magnetic field has been shown to induce subgap states as seen by an increase in spectral weight of low-energy fluctuations detected by inelastic neutron scattering experiments $[21,33,34]$.

A special point in the phase diagram is found at the anomalous 1/8 doping where both LSCO and LBCO show very different behavior compared to the neighboring doping regimes. This effect is particularly strong in LBCO, where bulk superconductivity is almost fully suppressed, with $T_{N} \sim$ $40 \mathrm{~K}$ and $T_{c}=5 \mathrm{~K}[16,35,36]$, although two-dimensional superconducting correlations still exist up to $40 \mathrm{~K}[35,37]$. LBCO also displays pronounced charge stripes with a periodicity of four lattice spacings [35], possibly related to a structural phase transition between the common cuprate low-temperature orthorhombic phase and a low-temperature tetragonal phase at a temperature close to the onset of charge order $[16,35]$. The stripe order is very pronounced in LBCO, spanning a doping range of $0.095<x<0.155$ [1,9,38]. Charge stripes have also been found in LSCO [39-41] and LCOO [42], both with hole concentration $\sim 1 / 8$.

For both LSCO and LBCO in the $1 / 8$ phase, magnetic fluctuations display a small, partial gap of the order $0.5 \mathrm{meV}$ ascribed to spin anisotropy. Both gap and fluctuation intensities are insensitive to an external magnetic field $[43,44]$. The absence of a full spin gap in LBCO has previously been interpreted as a deviation from uniform $d$-wave superconductivity toward a modulated phase of coexisting superconductivity and low-energy magnetic fluctuations in the form of a pair-density wave (PDW) [45]. The magnetic fluctuations continue up to about $200 \mathrm{meV}$ in an "hourglass" shape common for most cuprates $[1,8,46,47]$.

In LCOO, there is a strong tendency toward electronic phase separation [48]. Muon spin rotation experiments have been critical in demonstrating the spatial separation of the magnetic and superconducting phases [48,49]. Notable tools for characterizing each phase include neutron scattering [49-51], flux-pinning studies [52], resonant x-ray scattering [42], and scanning nano x-ray diffraction [53,54]. The intercalant oxygen ions take up interstitial sites separated by a regular number of unit cell layers in a process known as staging. The separation between intercalant layers, the stage number, is a rough measure of the overall doping. For instance, samples with $y \sim 0.06$ are known to exhibit $T_{c}=32 \mathrm{~K}$ and a stage-6 structure. Similarly, stage- 4 samples have $T_{c}=$ $42 \mathrm{~K}$ and periodicity 4 .

Neutron diffraction has shown strong static spin stripe signals in most LCOO samples, with a magnetic ordering temperature $T_{N} \approx T_{c}$ [48]. Early work on LCOO found that an applied magnetic field induces a significant enhancement of the neutron signal from the static stripes [13,50,55], an observation which is similar to LSCO [43] but unlike LBCO [56]. Our sample, however, does not show a field-enhanced static signal and this feature thus appears to be a reflection of the extent of the static magnetic phase present in a particular sample. The dynamic stripes in LCOO with $T_{c}=32 \mathrm{~K}$ [10] and $T_{c}=42 \mathrm{~K}$ (stage-4) [13] were previously addressed in neutron scattering studies. In Ref. [10], the IC signal from dynamic stripes at $\hbar \omega=2-4 \mathrm{meV}$ was observed both above and below $T_{c}$.

Here we revisit the low-energy spectrum of LCOO with the objective to test the universality of magnetic order and fluctuations, compared to its cousins LBCO and LSCO, including the response to different parameter variations, such as magnetic field and temperature. LCOO is remarkably different from LBCO, and to some extent to LSCO, in that the appearance of static stripes does not suppress $T_{c}$. It is therefore relevant to search for underlying similarities in other parts of their magnetic spectra. For this reason, we here present the results of a comprehensive study of static and dynamic spin stripes in LCOO.

Our main findings are the absence of a spin gap in the low-energy fluctuation spectrum in the superconducting state and the absence of a significant magnetic field effect both in the elastic and inelastic channels. Furthermore, our $x y z$ polarization analysis reveals that the static stripes do not have any preferential direction between the two in-plane tetragonal $a_{T}$ and $b_{T}$ axes in this system.

\section{EXPERIMENTAL METHOD}

The samples used throughout this study were prepared by growing stoichiometric crystals of $\mathrm{La}_{2} \mathrm{CuO}_{4}$ (LCO) at the Technical University of Denmark in an optical image furnace using the traveling solvent float zone technique [57]. After annealing and x-ray characterization, chosen crystals were super-oxygenated in an aqueous bath at the University of Connecticut. The resulting LCOO crystals were cut into pieces of 3-4 g each, suitable for neutron scattering experiments. Smaller pieces of the samples were used for magnetic susceptibility measurements which revealed a single superconducting transition temperature of $41.3 \pm 0.8 \mathrm{~K}$ (midpoint), indicating the presence of a unique superconducting phase [48], see Fig. 1(a). We have confirmed that this estimate is representative for the entire sample by measurements of the depolarization of the neutron beam induced by the trapped magnetic flux inside the sample. The details of the experiment and the data are presented in Appendix B.

The data discussed in this paper were acquired during several neutron scattering experiments carried out on the triple-axis spectrometers FLEXX [58,59] at the Helmholtz Center Berlin (HZB), IN12 [60,61], a Jülich Centre for Neutron Science (JCNS) instrument outstationed at the Institut Laue-Langevin (ILL) and ThALES [62-64], at the ILL. Results from one of the ThALES experiments were previously reported in another context in Ref. [65]. Each experiment followed the evolution of both the magnetic order and spin fluctuations under different conditions such as varying temperature (IN12 and the first Thales experiment, Th1, see Figs. 1 and 2), applied magnetic field (FLEXX, see Fig. 3) and in polarization analysis configuration in order to determine the in-plane orientation of the magnetic moments (second Thales experiment, Th2, see Fig. 4).

All instruments used for this project employ a velocity selector on the incident beam, before the monochromator, in order to remove second-order contamination. In addition, 
a cooled Be filter between sample and analyzer was used in the second part of the IN12 experiment and in the Th1 experiment to further reduce background. The instrument setup on IN12 contained vertically and horizontally focusing monochromators, leading to a relaxed in-plane $Q$ resolution of about $0.02 \AA^{-1}$ (for $k_{f}=1.5 \AA^{-1}$ ) and even broader resolution out of the scattering plane, where the stripe signal from cuprates is nearly constant $[13,66]$. In the Th1 experiment only vertical focusing was employed, leading to an enhanced in-plane momentum resolution by a factor $2(0.01$ $\AA^{-1}$ ) and relatively loose resolution along the $c$ direction. For the polarization analysis (Th2) the instrument configuration contained Heusler (111) monochromator and analyzer as well as an orange cryostat placed inside a cryopad module [67] for accurate control of the spin polarization.

The sample environments used were orange cryostats for the experiments performed at the ILL and a 15-T cryomagnet for the experiment performed at the HZB. For all experiments, the sample was aligned in the $a-b$ plane, enabling access to the $(h, k, 0)$ scattering plane. Throughout the paper, the orthorhombic notation is used where the size of the unit cell is $a_{o}=5.33 \AA, b_{o}=5.40 \AA, c_{o}=13.20 \AA$ and the antiferromagnetic reflection is found at $\mathbf{Q}_{\mathrm{AFM}}=(1,0,0)$. However, due to twinning commonly present in these samples, each scattering point is a superposition of $(h, 0,0)$ and $(0, k, 0)$ reflections [68], meaning that antiferromagnetic scattering is also observed at $\mathbf{Q}_{\mathrm{AFM}}=(0,1,0)$ without deviation of the spins from the orthorhombic $b$ axis [51]. It should be mentioned that we have observed elastic intensity at the AFM reflection and confirmed its magnetic origin by polarized neutron scattering. In a homogeneously doped sample, AFM order would not be expected. However, in oxygen doped samples phase separation into oxygen-rich and oxygen-poor regions [10] might result in a remnant AFM order in small (presumably undoped) parts of the crystal. From this point forward, when referring to magnetic order we allude to the incommensurate order generated by the spin stripe arrangement.

During all experiments the sample was cooled slowly $(1 \mathrm{~K} / \mathrm{min})$ in the temperature range $300 \mathrm{~K}$ to $100 \mathrm{~K}$, in order to prevent unwanted effects arising from quenched oxygen disorder [66,69]. Measurements were performed in the SC phase at $2 \mathrm{~K}$ and in the normal phase at $45 \mathrm{~K}$. In the experimental setup, we resolve all four IC magnetic peaks at $\mathbf{Q}_{\mathrm{IC}}=(1 \pm 0.125,0 \pm \delta, 0)$ which are resolution limited and we obtain a lower bound for the correlation length of $\xi \sim$ $70 \AA$. The data were acquired through scanning over one or two of the four peaks with energy transfers between 0 and $10 \mathrm{meV}$. We furthermore followed the temperature dependence of the intensity of the peaks at $0,0.3$, and $1.5 \mathrm{meV}$ using one- and three-point measurements. The same crystals in different coalignment combinations have been used for all experiments. The total masses of the samples used in each experiment were as follows: FLEXX $8.84 \mathrm{~g}$, IN12 $15.79 \mathrm{~g}$, and ThALES $3.44 \mathrm{~g}$ (one single crystal). In the FLEXX and IN12 experiments the crystals were coaligned to within $1.3^{\circ}$ and $2^{\circ}$, respectively. For this particular study, where magnetic scattering is in focus, the use of greater sample mass allowed us to overcome the counting time restrictions imposed by the small cross section of inelastic magnetic scattering. With an increased neutron flux at the sample position, such as is the case at ThALES, a lower sample mass can provide better signal-to-noise ratio, since the size of the beam hitting both the cryostat and the sample can be significantly reduced.

Another manner of improving the signal to noise ratio is to tune the size of the resolution ellipsoid by varying the wave vector of the scattered neutrons, $k_{f}$. By using a smaller value of $k_{f}$, we obtain a better energy resolution, which is preferable when studying low energy excitations, where tails from the elastic scattering can greatly contribute to the inelastic background signal. For this reason, we chose to employ different $k_{f}$ values $\left(k_{f}=1.1,1.2,1.5,1.55 \AA^{-1}\right)$ when measuring excitations of different energies across the two experiments (IN12 and Th1) which are combined in Fig. 2(c). The (elastic) energy resolution quantified by the full width at half maximum (FWHM) in the ThALES and IN12 experiments in these settings was determined using incoherent scattering to 0.044(1), 0.070(1), 0.186(1), and 0.239(2) meV, respectively. In the FLEXX experiment $\left(k_{f}=1.55 \AA^{-1}\right)$, the FWHM is $0.301(4) \mathrm{meV}$.

To account for the difference in experimental setup, we normalize the measured raw counts in three steps. First we normalize to the monitor count, as shown in Figs. 2(a) and 2(b), which is inversely proportional to the incoming wave vector $\left(k_{i}\right)$. Taking into account that the scattering cross section is also proportional to $1 / k_{i}$, we obtain a scattering signal per monitor, where the dependence of $k_{i}$ is normalized out [70]. In order to obtain the susceptibility $\chi^{\prime \prime}$ in units of $\mu_{B}^{2} \mathrm{eV}^{-1} \mathrm{Cu}^{-1}$ similar to the procedure performed in Ref. [71], we normalized to a low-energy acoustic phonon at $\mathbf{Q}=(2,0,0)$, for $k_{f}=1.5 \AA^{-1}$ at IN12 and for $k_{f}=$ $1.55 \AA^{-1}$ at Thales (Th1). Last, for the IN12 data taken at other outgoing wave vectors, we corrected for the change in resolution volume [70] using a factor proportional to the ratio between the resolution volume $\left(V_{f}\right)$ corresponding to $k_{f}=1.5 \AA^{-1}$ (at which the phonon was also measured) and the ones corresponding to the different $k_{f}^{\prime}$ values at which each data set was taken $\left(V_{f}^{\prime}\right)$. We have used the following simplified ratio where we assumed constant reflectivity of the analyzer for the various outgoing wave vectors:

$$
\frac{V_{f}}{V_{f}^{\prime}}=\frac{k_{f}^{3}}{\tan \left(\theta_{A}\right)} \frac{\tan \left(\theta_{A}^{\prime}\right)}{k_{f}^{\prime 3}},
$$

where $\theta_{A}$ and $\theta_{A}^{\prime}$ are the Bragg angles of the analyzer for the two $k_{f}$ values.

We note that in Fig. 2 we present the dynamic susceptibility at the peak position, $\chi^{\prime \prime}\left(\mathbf{Q}_{\text {peak }}, \omega\right)$, rather than the average over the Brillouin zone to obtain $\chi^{\prime \prime}(\omega)$. We use this approach because averaging requires knowledge of the signal throughout the Brilloun zone, and we did the measurements only as one-dimensional scans. In the Appendix C, we provide a rough estimate of $\chi^{\prime \prime}(\omega)$ for the Thales data, and compare the result of this analysis with LBCO measurements of Ref. [45]. In addition, Appendix $\mathrm{C}$ includes a detailed description of all the steps of the normalization process. The appeal of absolute normalization is the possibility to directly compare measurements performed with different sample mass on various instruments as well as a quantitative comparison between the magnetic susceptibilities of different compounds. 
We have used two fitting routines. For the temperature dependence (Fig. 2) we implemented a so-called Sato-Maki function, first proposed as a model of describing antiferromagnetic correlations in chromium and its alloys [72]. This was later comprehensively explained and used by Aeppli et al. to fit neutron scattering measurements of magnetic fluctuations in optimally doped LSCO [73]. While Aeppli et al. used tetragonal notation, we have converted the formalism to the orthorhombic structure. The associated lattice parameters $a_{o}=b_{o}=5.3 \AA$ have been used, since these are too close to distinguish from one another given the resolution of our inelastic neutron scattering experiments. The scattering amplitude is thus fitted to the function:

$$
S(Q, \omega)=\frac{[n(\omega)+1] \chi^{\prime \prime}(\omega, T) \kappa^{4}(\omega, T)}{\left[\kappa^{2}(\omega, T)+R(Q)\right]^{2}},
$$

where

$$
R(Q)=\frac{4\left[\left(Q-Q_{\mathrm{AFM}}\right)^{2}-\delta^{2}\right]^{2}}{\left(2 a_{0} \delta\right)^{2}} .
$$

Here $[n(\omega)+1]$ is the thermal population factor for downscattering (neutron energy loss), $\chi^{\prime \prime}(\omega, T)$ is the imaginary part of the dynamic susceptibility at the peak position, $\kappa(\omega, T)$ is the peak width, and $\delta$ is the incommensurability of the signal measured around the antiferromagnetic point $\left(\mathbf{Q}_{\mathrm{AFM}}\right)$. Note that the possible differences in incommensurabilities $\delta_{h}$ and $\delta_{k}$ are small compared to the width of the resolution function [65] and we simply set $\delta_{h}=\delta_{k}=\delta$ henceforth. This routine imposes equal amplitude, width, and incommensurability for the two peaks that are scanned over during the measurement. The incommensurability is defined as the distance between the peak center and $\mathbf{Q}_{\mathrm{AFM}}=(1,0,0)$.

To analyze the rest of the data we used a Gaussian model with constrained equal widths and incommensurability, because part of the data were obtained by scanning over a single peak (Fig. 3) or due to the appearance of additional parasitic scattering on top of one of the peaks (Fig. 4).

\section{RESULTS}

The sample exhibits a single and clean superconducting transition at $T_{c}=42 \mathrm{~K}$, as seen from the temperature dependence of the diamagnetic signal shown in Fig. 1(a) and confirmed by depolarization measurements. This supports the presence of a single dominant staging structure which, given its critical temperature, was previously found to correspond to stage-4 samples $[10,68]$. The temperature dependence of the magnetic order in Fig. 1(b) unveils that the onset temperature of the elastic stripe signal coincides, within errors, with the onset of superconductivity, $T_{c} \approx T_{N}$.

The low-energy magnetic fluctuations have a much higher onset temperature than superconductivity $\left(T_{\text {onset }}>70 \mathrm{~K}>\right.$ $T_{c}$ ) and do not show any dramatic signatures at $T_{N}$ or $T_{c}$, see Fig. 1(c). At the lowest energy $\hbar \omega=0.3 \mathrm{meV}$, a broad increase in intensity is observed around $T=30 \mathrm{~K}\left(<T_{c}\right)$. A similar temperature dependence of fluctuations of slightly higher energy ( $\hbar \omega=2 \mathrm{meV})$ was observed by Lee et al. [13] in a stage-4 LCOO sample. These measurements showed an increase in intensity down to $\sim 30 \mathrm{~K}$, followed by a less pronounced suppression at lower temperatures. In stage-6
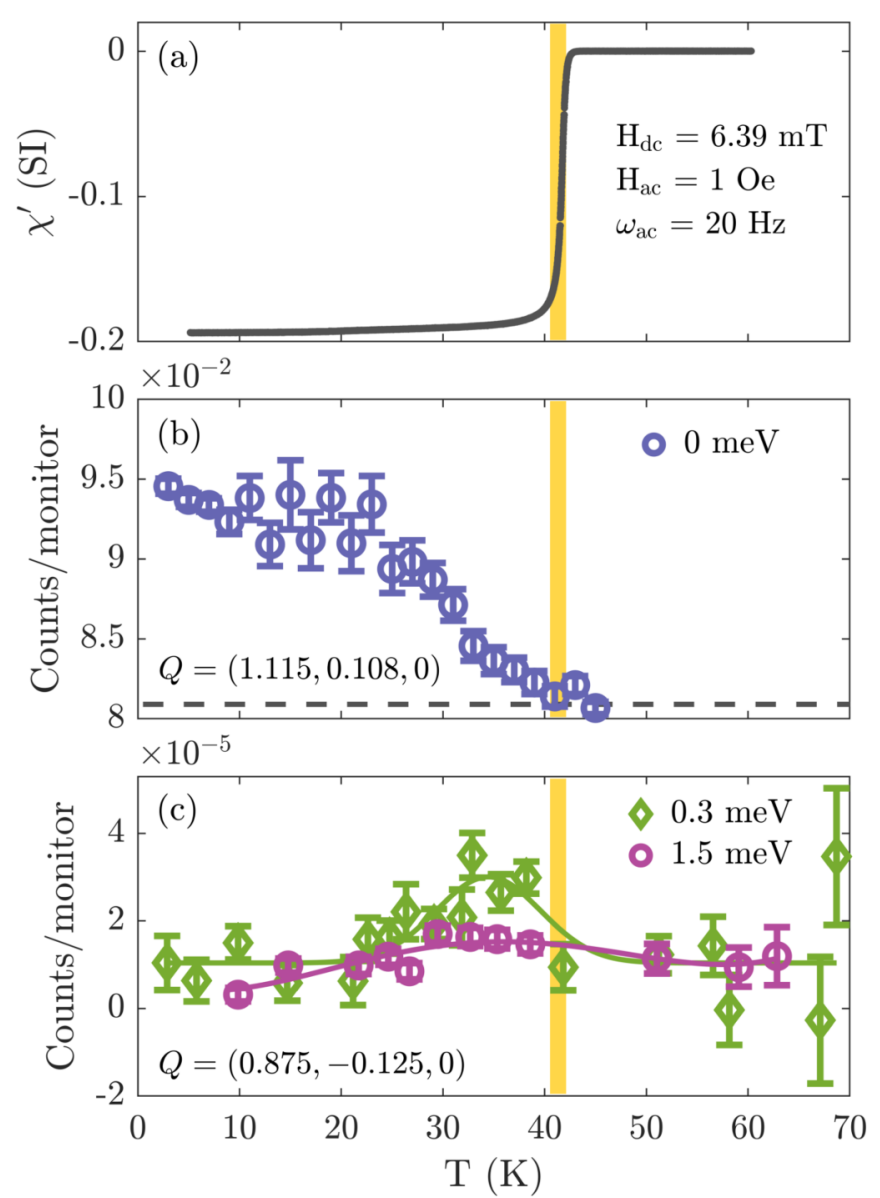

FIG. 1. (a) The real part of AC magnetic susceptibility, $\chi^{\prime}$, showing the sharp transition to a diamagnetic state on cooling below the superconducting critical temperature. $T_{c}$ is indicated by the yellow stripe which spans a temperature range $41.3 \pm 0.8 \mathrm{~K}$. The steps followed in the conversion from emu to SI units are presented in Appendix A. (b) Temperature dependence of magnetic order measured as 1-point scans on top of one of the IC peaks $(1.115,0.108,0)$. The incoherent background has not been subtracted. The dashed line indicates the background level extracted from an energy scan taken away from the incommensurate peak position, at $(1.1,-0.24,0)$. (c) Temperature dependence of magnetic fluctuations with energy 0.3 and $1.5 \mathrm{meV}$. Data were measured as three-point scans with $k_{f}=1.2 \AA^{-1}$ $[\Delta E=0.070(1) \mathrm{meV}]$ and the intensity is obtained as the difference between the point on top of the peak, at $(0.875,-0.125,0)$, and the average of the two background points.

samples, some of us earlier reported [10] the same temperature behavior of low-energy magnetic fluctuations $(2-4 \mathrm{meV})$. In that work, the peak in intensity happened to coincide within errors with the critical temperature $T_{c}=31 \mathrm{~K}$ and to follow the same trend as underdoped $(x=0.12)$ LSCO samples of similar $T_{c}$ [43]. The present data show that the increase in intensity of the low-energy fluctuations at temperatures close to $30 \mathrm{~K}$ is likely a universal feature of LCOO and underdoped LSCO superconductors regardless of their corresponding superconducting critical temperatures.

We now address how cooling below $T_{c}$ influences the low-energy spectrum in the energy range $\hbar \omega=0.3-10 \mathrm{meV}$. Figures 2(a) and 2(b) show examples of representative scans 

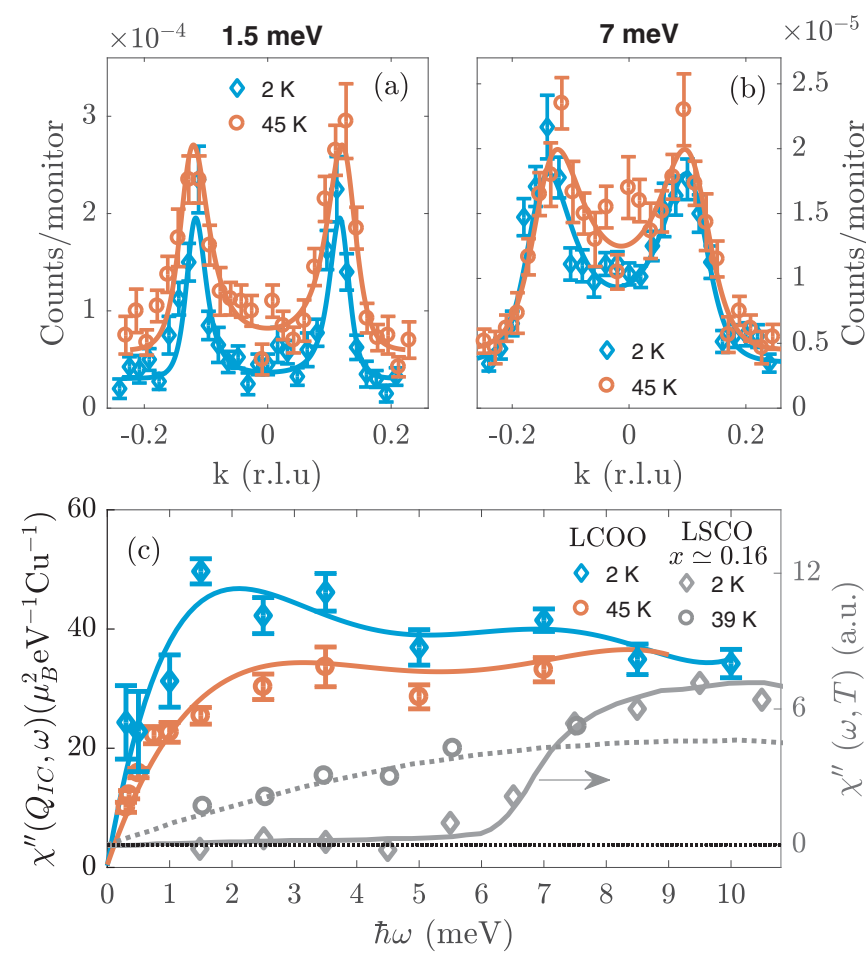

FIG. 2. [(a) and (b)] Representative inelastic scans through the incommensurate positions $( \pm \delta, 1-\delta, 0)$ where $\delta=0.125$, collected at 1.5 and $7 \mathrm{meV}$ with fixed outgoing wave vectors $k_{f}=1.55 \AA^{-1}$ and $k_{f}=1.5 \AA^{-1}$, respectively. The data were collected at ThALES (a) and IN12 (b) at two temperatures: Blue diamonds show data in the superconducting state $(2 \mathrm{~K})$ and orange circles show data within the normal state $(45 \mathrm{~K})$. The solid lines are Sato-Maki function fits to the raw data as described in the text. (c) Dynamic susceptibility $\chi^{\prime \prime}\left(\mathbf{Q}_{\text {IC }}, \omega\right)$ measured inside ( $2 \mathrm{~K}$, blue) and outside (45 K, orange) the superconducting dome. Solid lines are guides to the eye. Each data point represents an average of values obtained at different $k_{f}$ values and on different instruments (ThALES and IN12). In gray symbols, we show for comparison the magnetic susceptibility measured inside and outside the superconducting state by Lake et al. [33] on a optimally doped $x=0.163$ LSCO sample.

taken at Thales and IN12, respectively, at temperatures both inside $(2 \mathrm{~K})$ and outside $(45 \mathrm{~K})$ the superconducting phase. The apparent increase in integrated intensity at the low energies as a function of increased temperature [Fig. 2(a)] is merely an effect of the Bose occupation factor. Figure 2(c) depicts the imaginary part of the dynamical spin susceptibility $\chi^{\prime \prime}\left(\mathbf{Q}_{\mathrm{IC}}, \omega\right)$, expressed in units of $\left(\mu_{B}^{2} \mathrm{eV}^{-1} \mathrm{Cu}^{-1}\right)[70,71]$. The data demonstrate that spin fluctuations with energy $\hbar \omega>$ $4 \mathrm{meV}$ are rather insensitive to the onset of superconductivity. At lower energies, cooling below $T_{c}$ leads to an increase in the spin susceptibility at $\mathbf{Q}_{\mathrm{IC}}$. This is unlikely to be a direct consequence of the onset of superconductivity but clearly shows that there is no tendency toward a suppression of the low-energy magnetic spectrum in the superconducting phase. The sample thus shows no evidence of a spin gap, although a small partial gap at $\hbar \omega \sim 0.5 \mathrm{meV}$ cannot be excluded.

Our observations are in contrast to studies of optimally doped LSCO, with a similar high value of $T_{c}$, where the superconducting transition is accompanied by the opening
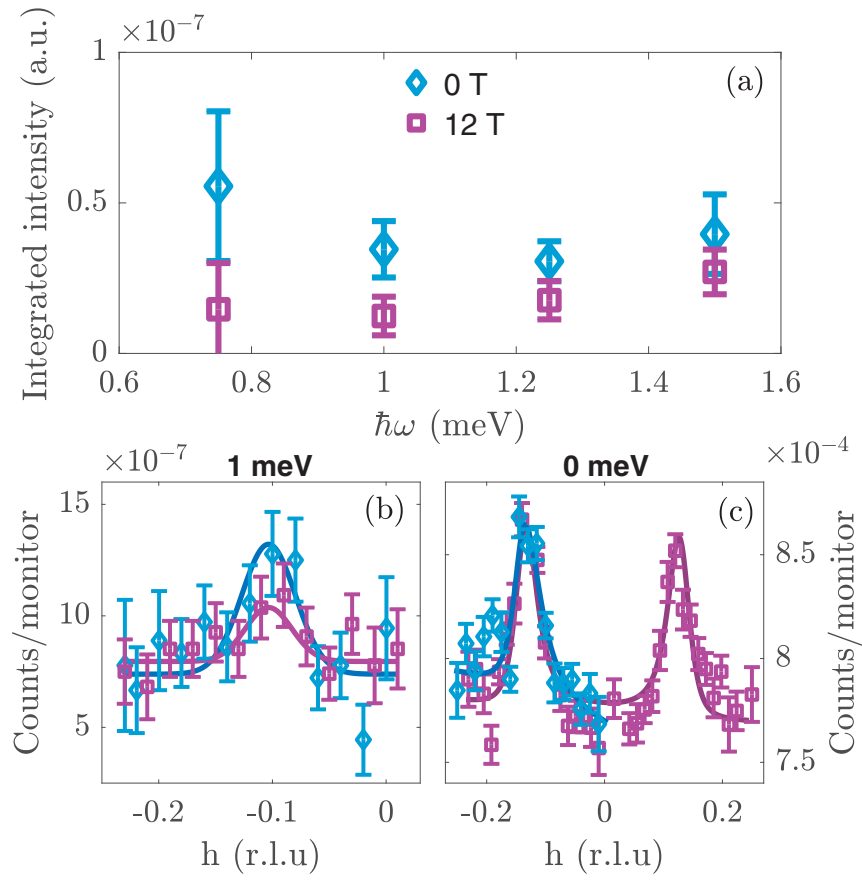

FIG. 3. (a) Magnetic field dependence of low-energy spin excitations measured at $2 \mathrm{~K}$. Representative (b) inelastic constant energy scan $(\hbar \omega=1 \mathrm{meV})$ and (c) elastic scans collected in the superconducting phase $(2 \mathrm{~K})$ with and without applied magnetic field. The solid lines are Gaussian fits to the raw data.

of a clean spin gap in the low-energy magnetic spectrum [11,33]. Subsequently, an increase in temperature above $T_{c}$ induces subgap states. To visualize this difference, we plot in Fig. 2(c) the data obtained in this sample together with the data of LSCO $x=0.163$ from Ref. [33]. On the other hand, the low-energy spectrum bear close resemblance to LBCO [45] and LSCO $x=0.12$ [43] despite the higher $T_{c}$ and the tendency toward phase separation not being observed in the latter compounds.

In order to gain a more comprehensive understanding of the magnetism of our oxygen-doped sample, we have also pursued the effect of an applied magnetic field. This experiment was performed on the FLEXX spectrometer, and since measurements were acquired mostly as scans over one of the incommensurate peaks, a single Gaussian function was used to fit the data. In addition, no conversion to absolute units was carried out because the temperature was kept constant and the energy difference produces a negligible effect in this regime. Along the $c$ axis, a $H=12 \mathrm{~T}$ magnetic field was applied, which is much lower than the upper critical field $H_{c 2} \sim 60 \mathrm{~T}$ (see Ref. [74]). The data shown in Fig. 3 illustrate the lack of response in the elastic channel and a small decrease with field in the low-energy inelastic channel. The insensitivity of magnetic order to an applied magnetic field contradicts reports from the literature on LCOO. We will comment on this aspect in Sec. IV C.

To access the orientation of the magnetic moments in static and dynamic magnetic stripes, we employed polarized neutron scattering analysis with three different spin configurations of the incoming neutron beam. First, the neutron 
TABLE I. Expected components of the magnetic correlation function, $M$, measured in different spin configurations of the incoming neutron beam, $S$. In this table, $M_{\alpha \alpha}=$ $\frac{1}{2 \pi \hbar} \int d t e^{i \omega t}\left\langle M_{\alpha}^{\dagger}\left(\mathbf{Q}_{\mathrm{IC}}, 0\right) M_{\alpha}\left(\mathbf{Q}_{\mathrm{IC}}, t\right)\right\rangle$, and $M_{\alpha}\left(\mathbf{Q}_{\mathrm{IC}}, t\right)$ is the spin component $(\alpha=x, y, z)$ at the incommensurate position at time $t$. We have assumed that there is no chiral contribution to the $S \| x$ channel. Contributions from incoherent scattering and nuclear coherent scattering are omitted for simplicity as these would give rise to the same background within all the spin channels. A more detailed overview of the formalism involved can be found, e.g., in Refs. [75,76].

\begin{tabular}{lcc}
\hline \hline Spin direction & NSF & SF \\
\hline$S \| x$ & 0 & $M_{y y}+M_{z z}$ \\
$S \| y$ & $M_{y y}$ & $M_{z z}$ \\
$S \| z$ & $M_{z z}$ & $M_{y y}$ \\
\hline
\end{tabular}

polarization was chosen parallel to the scattering vector $\mathbf{Q}$, defined as the $x$ direction in the following. In this set-up, we performed scans in both the spin flip (SF) and the non-spinflip (NSF) configurations. Afterwards, only the SF channel was measured with the neutron spin aligned along the $y$ and $z$ directions, $z$ being out of the scattering plane. This $x y z$ polarization analysis allowed us to determine the contribution of different components of the magnetic order in the sample, as illustrated in Table I.

In the elastic channel, Fig. 4(b), we observe a lack of intensity in the $S \| y$ spin flip channel and equal intensity in the other two SF channels. Compared to Table I, this provides definite evidence that $M_{z z}$ is (close to) zero and thus that the spin structure in the elastic channel resides in the $a-b$ plane. The spin direction of the parent compound is along the orthorhombic $b$ axis [51], and a previous report found that this is preserved on doping [13]. We therefore assume that the spins are aligned along the $b$ axis in our sample. This assumption becomes important in the discussion presented in Sec. IV A, where we attribute the IC signals to different twin domains and stripe orientations.

The inelastic polarized data of Fig. 4(a) shows scattering intensity in all spin channels. This means that there is an out-of-plane spin component to the scattering signal, which is expected both in the case of isotropic spin fluctuations, and in the limit of purely transverse fluctuations connected to the static SDW signal. In Sec. IV A we discuss the polarized measurements in further detail.

\section{DISCUSSION}

\section{A. Stripe structure and twinning}

To analyze the structure of the spin stripe modulation, we consider two arrangements of the charge stripes which are assumed to accompany the magnetic stripes as antiphase domain walls [42]. The two possible arrangements of charge stripes along the tetragonal axes are depicted in Fig. 5(c). To access information about the structure of the spin stripe modulation we need to take into account all four possible twin orientations, which are naturally present in the system due to its weak orthorhombicity. We follow the detailed description of the twinning pattern presented in Ref. [77] (note that the
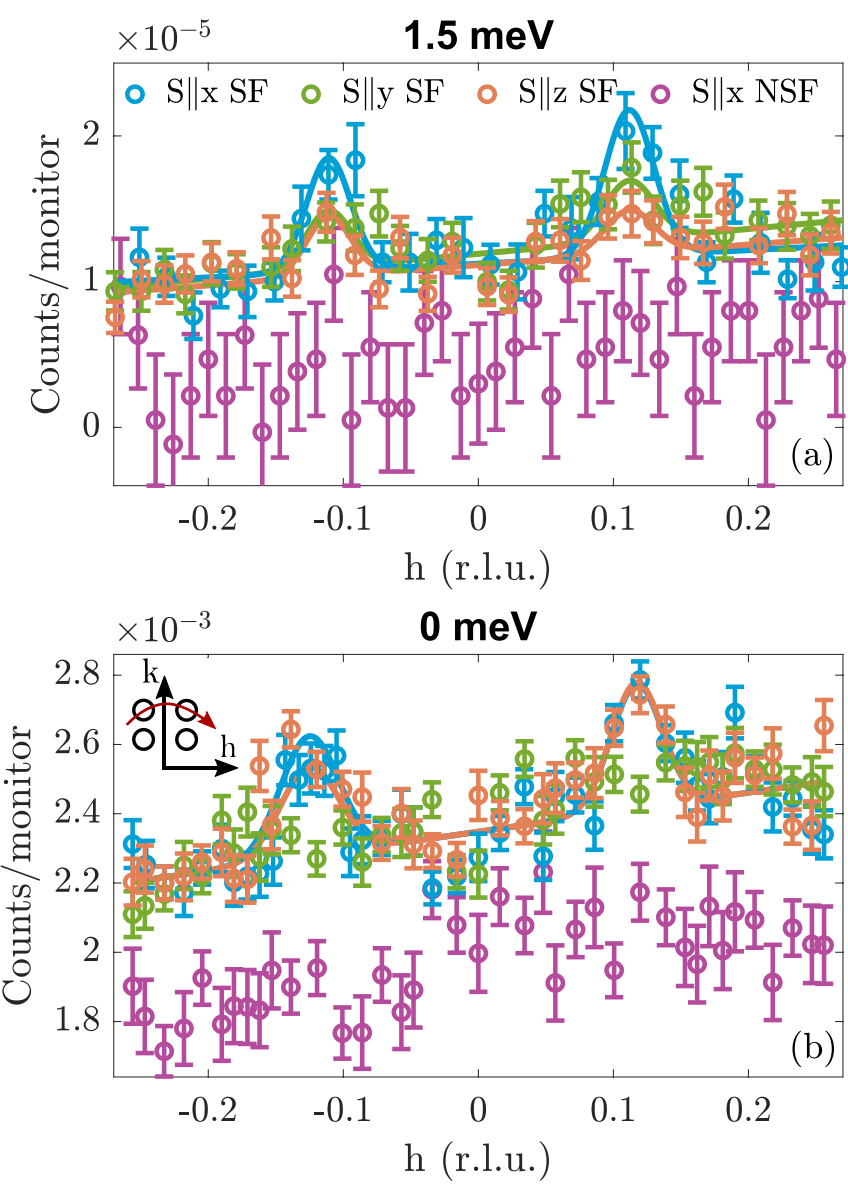

FIG. 4. (a) Inelastic constant energy scan $(\hbar \omega=1.5 \mathrm{meV})$ and (b) elastic scans collected in the superconducting phase (30 K and $5 \mathrm{~K}$, respectively) in SF, with three spin configurations, and NSF mode. The scan direction is represented in the inset of subplot (b). In panel (a) the NSF data are displaced by a factor $-0.7 \times 10^{-5}$ for better visualisation. $S$ denotes the incoming neutron beam spin direction. The solid lines are Gaussian fits to the raw data.

tetragonal notation in the cited reference corresponds exactly to our orthorhombic cell). This twinning structure complicates the interpretation of the data significantly. We present a detailed discussion based on the electronic stripe structure combined with the crystal twinning and illustrate the different contributions in Fig. 5. The typical twinning pattern of peaks along the measured $(0, k, 0)$ direction is depicted in Fig. 5(b), where the orthorhombicity is exaggerated for clarity. The spin direction is drawn along the orthorhombic $b$ axis of the local coordinate system for each of the four twin peaks.

As mentioned in the Introduction, the presence of magnetic stripes, which are in general incommensurate with respect to the crystal structure, is observed in neutron scattering experiments as a splitting of the antiferromagnetic $(0,1,0)$ reflection into four peaks. Signals along one diagonal, e.g., $\mathbf{Q}=(\delta, 1+\delta, 0)$ and $\mathbf{Q}=(-\delta, 1-\delta, 0)$, belong to charge stripe patterns along the tetragonal $a_{T}$ axis, while the signals along the other diagonal arise due to stripe formation in the perpendicular direction, i.e., the $b_{T}$ axis. The orientation of the charge stripe patterns, either along the tetragonal $a_{T}$ or $b_{T}$ 

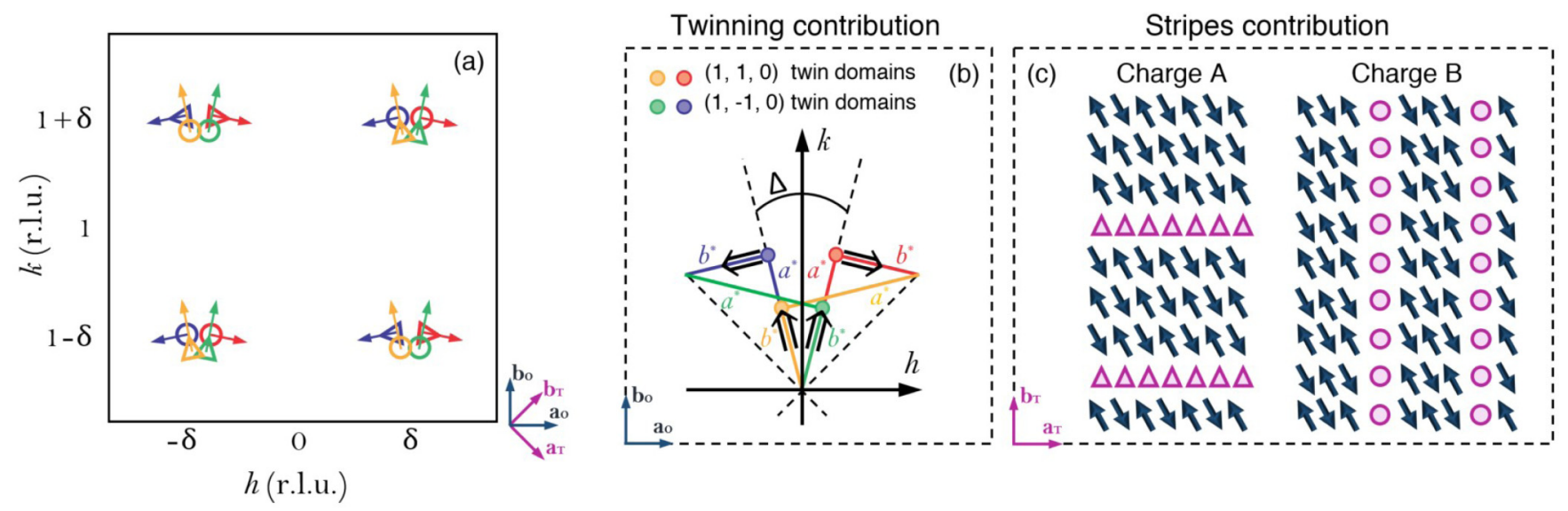

FIG. 5. (a) A cartoon illustration of the neutron scattering signal generated by the incommensurate AFM spin structure including contributions from all four crystal twins. (b) Twinning of a structural peak into two domains each composed of a pair of twins. The direction of the orthorhombic $b$ axis, which corresponds to the spin direction, is shown by a double-arrow for each twin peak. $\Delta$ is the angular separation between two twin reflections [77] and in our orthorhombic system has a value $\Delta=90^{\circ}-2 \arctan \left(\frac{a}{b}\right) \sim 0.8^{\circ}$, a splitting too small to be resolved given our $Q$ resolution. $a^{*}$ and $b^{*}$ are the reciprocal lattice constants in orthorhombic notation. Image adapted from the Supplemental Material of Ref. [65]. (c) The stripe pattern arising from charge stripes running along the tetragonal $a_{T}$ axis (Charge A) and the tetragonal $b_{T}$ axis (Charge B). The triangles and circles indicate how the charge orientations corresponds to the incommensurate peak structures in (a) for the local coordinate system of each twin type, which are rotated by $90^{\circ}$ with respect to each other.

axis, are illustrated in Fig. 5(c). The commensurate structure shown here is the special case of a peak splitting of $\delta=1 / 8$.

In the most general case, we expect the twinning structure to be present at all four peaks in the incommensurate peak quartet, as suggested by the color-coded symbols in Fig. 5(a). This figure furthermore provides an illustration, through the two types of symbols, of the charge stripe orientation that underlies each peak structure. Charge stripes along the tetragonal $a_{T}$ axis are depicted by triangles, while charge stripes along the tetragonal $b_{T}$ axis are shown by circles with reference to Fig. 5(c). Because each twin domain is composed of a pair of twins with interchanged $a_{O}$ and $b_{O}$ axes, we obtain two sets of twins with axes oriented in the same direction, namely red/blue and yellow/green. For the yellow/green domains, stripes along the $a_{T}$ axis (charge A) will give incommensurate magnetic peaks at positions $\mathbf{Q}=(\delta, 1+\delta)$ and $\mathbf{Q}=(-\delta, 1-\delta)$, while stripes along the $b_{T}$ axis (charge B) will cause incommensurate magnetic peaks along the other diagonal, i.e., at $\mathbf{Q}=(-\delta, 1+\delta)$ and $\mathbf{Q}=(\delta, 1-\delta)$. The situation is circumvent for the red/blue domains, because the $a_{T}$ and $b_{T}$ axes are interchanged in these twins compared to the green/yellow twin domains, as illustrated in Fig. 5(b).

In this way, the underlying charge structure for each twin contribution to the incommensurate signal is shown as circles and triangles in Fig. 5(a) with the color code as defined in Fig. 5(b). The spin directions for each peak is depicted by arrows, showing the direction of the orthorhombic $b$ axis of each domain.

In the experiment, we scanned over peaks belonging to both types of charge stripes by scanning through $\mathbf{Q}=$ $(-\delta, 1+\delta)$ and $\mathbf{Q}=(\delta, 1+\delta)$. The scanning direction is shown in the inset of Fig. 4(b). Since we only register signals where the scattering vector is perpendicular to the spins, we primarily pick up intensity due to the red/blue domains and only negligible weight from the yellow/green domains. Thus, we compare the relative strength of the charge types A and B of the red/blue twins and we can decide whether the system displays charge stripes along only one of the tetragonal axes or along both directions.

As shown in Fig. 4(b), we find that the peaks at $\mathbf{Q}=$ $(-\delta, 1+\delta)$ and $\mathbf{Q}=(\delta, 1+\delta)$ have similar amplitudes. From the equal signal amplitudes at both incommensurate positions, we conclude that charge stripes form along both the $a_{T}$ and $b_{T}$ direction and are equally present within the sample. In the event that charge stripes had a preferred direction (parallel or perpendicular to the $b_{T}$ axis), only one of the two IC peaks would have been visible. Our finding that the system displays charge stripes along both tetragonal axes is similar to the observation in LBCO with $x=\frac{1}{8}$ [5]. However, our data do not provide information about a possible orthogonal arrangement of stripes in adjacent planes, as discussed in the case of LBCO [17] and most likely, phase sensitive measurements are required to pursue this further.

We now turn to the inelastic polarized data of Fig. 4(a). As opposed to the static signal, which shows that spins are purely in-plane, the inelastic scattering signal at $\hbar \omega=$ $1.5 \mathrm{meV}$ shows a clear out-of-plane spin component. First, we consider the expected outcome of the signal ratios in the case of isotropic spin fluctuations, where fluctuations along all three spin directions are equal in magnitude, i.e., where the transverse and longitudinal fluctuations are equally strong. Then we would expect to see the same signal strength in the spin channels $S \| y$ and $S \| z$ and double intensity strength in the spin channel $S \| x$, i.e., a signal ratio of $2 / 1 / 1$ for the spin channels $x / y / z$. At the opposite end, we consider isotropic transverse fluctuations with longitudinal fluctuations being negligible, i.e., transverse fluctuations of equal strength in all directions perpendicular to the spin direction, but no fluctuations in the direction of the ordered moment. In this case, we would expect a signal ratio of $3 / 2 / 1$ taking into account the twinning structure. To distinguish between these two limiting cases, we calculate the ratio between the peak 
TABLE II. Comparison of the fitted intensity of the inelastic polarized neutron data. The ratio of intensity in the different spin channels is shown in terms of peak area. The values are obtained by adding the fitted parameters of the two peaks. For the fits, the peaks position and widths are fixed to the values obtained by fitting all the data (from all three channels) combined. The last two columns show the expected signal ratios in the case of isotropic fluctuations and purely transverse fluctuations.

\begin{tabular}{lcccc}
\hline \hline $\begin{array}{l}\text { Neutron spin } \\
\text { direction }\end{array}$ & $\begin{array}{l}\text { Magnetic } \\
\text { scattering }\end{array}$ & Area & Isotropic & Transverse \\
\hline$\frac{S \| x}{S \| y}$ & $\frac{M_{y y}+M_{z z}}{M_{z z}}$ & $2.2 \pm 0.7$ & $2 / 1$ & $3 / 2$ \\
$\frac{S \| x}{S \| z}$ & $\frac{M_{y y}+M_{z z}}{M_{y y}}$ & $2.5 \pm 0.8$ & $2 / 1$ & $3 / 1$ \\
$\frac{S \| y}{S \| z}$ & $\frac{M_{z z}}{M_{y y}}$ & $1.1 \pm 0.4$ & $1 / 1$ & $2 / 1$ \\
\hline \hline
\end{tabular}

areas of the three spin channels with the results shown in the third column of Table II. These results are compared to the expected ratios in the case of isotropic and purely transverse fluctuations, see fourth and fifth columns of Table II. This provides guidance to the dominating nature of the incommensurate spin fluctuations at $1.5 \mathrm{meV}$.

The data indicates that the spin fluctuations are isotropic, in particular because the signals with polarization along the $y$ and $z$ directions are almost equal. However, we have only taken into account the extreme cases. It should be noted that intermediate cases could lead to similar signal ratios. For example, anisotropic transverse fluctuations with a more pronounced in-plane component (in the $x-y$ plane) than out-ofplane component could also result in a signal ratio of $2 / 1 / 1$ for the spin channels $x / y / z$ which would be compatible with the obtained signal ratios. A detailed study of fluctuations, with measurements also along the $l$ direction in reciprocal space has the potential to shed light onto this matter. Due to long counting times, imposed by the polarization set-up and the intrinsic weak magnetic inelastic signal, combined with the necessity to realign the sample, we have not been able to further pursue this idea.

Another perspective on the relation between static order and low-energy spin fluctuations in the very same sample was acquired recently by some of us in Ref. [65]. A difference in incommensurability between the static signal and low-energy spin fluctuations led us to conclude that magnetic order and fluctuations likely originate from separate spatial domains within the crystal. If the nature of the low-energy spin fluctuations had been primarily transverse, i.e., spin-wave-like Goldstone modes of the static spin order, then this would have contradicted the findings of Ref. [65]. The observation that the $1.5 \mathrm{meV}$ fluctuations are more likely of isotropic character supports the interpretation in Ref. [65] and reaffirms our hypothesis that the low-energy fluctuations reside in different parts of the crystal than the static order. These regions could very well be where superconductivity is present, as we will discuss in the next section.

\section{B. Phase separation and intertwined orders}

Figure 1 demonstrates that the sample exhibits similar critical temperatures for superconductivity and magnetic order, i.e., $T_{c} \simeq T_{N}$. This finding is supported by results from local probe nuclear magnetic resonance (NMR) measurements [78] and was also found by neutron scattering [13,69] in LCOO samples with the same critical temperature as the one used in this study. While this could be interpreted as a microscopic coexistence of magnetic and superconducting order [69], there is also the possibility of a microscopic phase separation of the crystal into different domains where each contains only one type of order, either magnetic or superconducting, both phases with comparable free energy [49].

Evidence of phase separation was found in the local probe NMR and $\mu$ SR studies of similar systems $[48,49,78,79]$ and in a neutron scattering study of this very same sample [65]. Taking this into account, some of us have previously advocated for a phase separation in which one part of the crystal shows static magnetic order and associated Goldstone modes, while other parts of the crystal display low-energy fluctuations without static order at a slightly different incommensurability. We speculate that the Goldstone modes associated with the static magnetic order are too weak to be detected with neutrons when superimposed on the signal from the low-energy fluctuations.

Another proposal of phase separation in the cuprate family was put forward in the case of underdoped LSCO [11]. There, the electronic structure of the sample was interpreted as divided into two phases; magnetic order with low-energy fluctuations $(<4 \mathrm{meV})$ in some parts of the crystal and gapped higher energy fluctuations and superconductivity in other parts of the crystal. Evidence for this phase separation was (a) a dip in the energy spectrum at $\sim 4 \mathrm{meV}$ and (b) a small change of linewidth of the signal at this energy. We do not observe neither a dip nor an abrupt change of linewidth as a function of energy. However, we note that such effects are very subtle and hard to detect, and we therefore hesitate to draw definite conclusions based on our observations.

In the large number of studies performed on the $\mathrm{La}_{2-x} \mathrm{M}_{x} \mathrm{CuO}_{4+y}$ family of superconductors, we observe two general types of magnetic behavior in the superconducting state: (1) compounds that do not exhibit magnetic order and have gapped low-energy spin excitations below $T_{c}$ and (2) compounds where magnetic order is present concomitantly with a continuous spectrum of spin fluctuations, albeit with reduced strength at low energies. Our stage-4 LCOO sample belongs to the latter category, exhibiting both static stripe order as shown in Fig. 4(b) and ungapped low-energy fluctuations as displayed in Fig. 2(b). We note that fluctuations appear insensitive to the onset of superconductivity. In fact there is a strengthening of the low-energy spin susceptibility at the lowest temperatures. The suppression of the very lowenergy signal $(\hbar \omega<4 \mathrm{meV}$ ) with increasing temperature is likely a consequence of decreased spin coherence. Figure 1(c) points to a complete destruction of spin coherence at $T \sim$ $70 \mathrm{~K}$. Our sample is therefore different from optimally doped LSCO, which does not show static order and in which lowenergy spin fluctuations are gapped in the superconducting state and furthermore persist up to $350 \mathrm{~K}$ [73]. The latter feature is depicted by the gray data points in Fig. 2(b) which are reproduced from Refs. [20,33]. At doping $x \simeq 0.16$, LSCO exhibits a spin gap of roughly $\Delta_{0.16} \sim 6-7 \mathrm{meV}$. This sample has a similar optimal value of $T_{c} \simeq 39 \mathrm{~K}$ as found in LCOO. 
In the event that LCOO had a (magnetically) similar superconducting phase as in optimally doped LSCO, this would show up as an incomplete spin gap around $\Delta_{0.16}$ in our measurements. This is clearly not the case.

We can think of four scenarios that would be compatible with the lack of spin gap: (1) if superconductivity arises in yet other parts of the crystal, where there are no low-energy spin fluctuations of $\hbar \omega<7 \mathrm{meV}$, (2) an increase in the low-energy fluctuations in the nonsuperconducting part of the sample exactly matches a decrease of the fluctuations in the superconducting regions, (3) a spin gap emerges at other wave vector not explored in this study, or (4) superconductivity coexists with and does not compete with low-energy magnetic fluctuations. The two first scenarios seem unlikely, since (1) would imply regions with a very different low-energy electronic behavior not observed in any other superconductors to date, (2) would seem like an improbable coincidence, and (3) time of flight neutron measurements on similar samples [32,45], which explored larger portions of the reciprocal space, have not shown any other contributions to magnetic scattering emerging at a different wave vectors. Thus, we are left with the fourth scenario, which points to a coexistence of low-energy fluctuations and superconductivity in LCOO.

In underdoped LBCO, a lack of spin gap has also been observed and interpreted as evidence of a PDW type of electronic structure with intertwined modulated superconducting order and spin stripes [45,80]. In our sample, we interpret the absence of any significant decrease in $\chi^{\prime \prime}$ for $T<T_{c}$ at energies $\hbar \omega \leqslant 10 \mathrm{meV}$, as an indication that superconductivity in LCOO microscopically coexists with the low-energy magnetic fluctuations. However, because of additional evidence that the low-energy fluctuations do not occur in the same spatial regions as the static magnetic order [65], this proposed coexistence phase is different from the usual PDW phase: Only low-energy fluctuations are intertwined with superconductivity, while magnetic order appears as a separate, probably competing, phase. In addition, by presenting our data on an absolute scale, we find that the magnetic spectral weight of low-energy fluctuations in LCOO is approximately five times lower than that of LBCO or the parent compound, LCO (see Appendix C). In LBCO, the strong magnetic response was taken as indication that magnetic order and fluctuations coexist locally with superconductivity in the entire bulk of the sample. The reduction of magnetic spectral weight in LCOO found here points to the fact that there is a smaller volume fraction attributed to each of the different electronic phases. In particular, a fraction of the sample shows AFM order and the corresponding magnetic signatures are not included in our analysis, leading to an apparent reduction in the spin susceptibility when presented in absolute units.

The temperature dependence of the magnetic susceptibility of LSCO with $x=1 / 8$ doping [43] is remarkably similar to our findings in LCOO. This might indicate a similar coexistence of low-energy fluctuations and superconductivity in that system as well. Note that an additional similarity of LCOO and LSCO $x=0.12$ is the curious insensitivity to a magnetic field of the low-energy fluctuations, which could provide further support for a coexistence phase.

To further elucidate the nature of the superconducting state, it would be interesting to investigate the presence or absence of a magnetic resonance peak by inelastic neutron measurements of high energy spin excitations. One of the fingerprints of the PDW is the absence of the magnetic resonance peak $[45,80]$ otherwise observed, in standard $d$-wave SC, at $E_{\text {cross }} \approx 40-50 \mathrm{meV}[8,47,81]$.

\section{Magnetic field effect}

A uniform $d$-wave superconductor is expected to show magnetic field-enhancement of the magnetic spectral weight at low energies since the destruction of superconductivity in the vortex cores paves the way for magnetic order [22]. This is opposite to our observations in LCOO, see Fig. 3(a) where we instead of a field-enhancement find a small suppression of the spectral weight at low energies in the range $\hbar \omega=0.7-1.5 \mathrm{meV}$. In optimally doped LSCO samples, the subgap field-induced states are thought to originate from a separate electronic phase most likely prevailing in the vicinity of the vortices [33,82]. This explanation is similar to the above-mentioned phase separation scenario proposed by Kofu et al. [11] where low-energy fluctuations, in underdoped LSCO samples, are thought to originate from a phase different from the superconducting one. In our sample, on the contrary, we argue that the low-energy magnetic fluctuations coexist with superconductivity without being in direct competition. For this reason, we do not expect a significant effect, on the magnetic excitations spectrum, induced by the appearance of a vortex lattice in the superconducting regions. This is in agreement with our observations of a very weak suppression of the low-energy signal in a 12-T applied magnetic field.

On the other hand, in terms of elastic stripes, in opposition to the lack of field effect in our sample [see Fig. 3(c)], Khaykovich et al. [50,55] and Lee et al. [69] have previously reported a significant field enhancement of the elastic signal, comparable to the effect of electronic disorder induced by quenched cooling, in both stage-4 and stage-6 LCOO samples. Taking into account the high quality of our crystal, which exhibits a sharp single transition to superconductivity at $42 \mathrm{~K}$ [Fig. 1(a)], we can think of two possible explanations for this discrepancy: (1) The slow cooling procedure we used in our experiments (with a cooling rate of $1 \mathrm{~K} / \mathrm{min}$ ) could differ from that used previously and result in a different arrangement of the excess oxygen, leading to a different electronic configuration at base temperature; (2) subtle differences in the oxygenation procedure could have created samples with different ground states containing various ratios of superconducting and magnetically ordered phases.

With respect to the first explanation, the exact cooling rate used during the field experiments is not stated in the literature. The only value we can compare against is the quench cooling rate of $\sim 2.8 \mathrm{~K} / \mathrm{min}$ [69] that creates a significant structural distortion which in turn induces an enhancement of the magnetic order. Only if our sample were to be in a highly disordered phase, following the cooling procedure, the effect of an applied magnetic field would be as insignificant as the one we have measured. This is highly unlikely, since our much lower cooling rate of $1 \mathrm{~K} / \mathrm{min}$ is expected to give rise to an ordered oxygen lattice at low temperatures.

The second and more likely scenario is based on observations by Chang et al. [82] demonstrating that the effect of 
an applied magnetic field is to enhance the magnetic order in LSCO samples toward a common plateau level, which corresponds to the ground state of systems with $1 / 8$ doping value. In this case, the initial fraction of the magnetically ordered phase in the sample dictates the magnitude of the impact of an applied magnetic field. Thus, the lack of a field effect which we have measured, indicates that our sample, in contrast with the ones used by Khaykovich et al. and Lee et al., exhibits a fully developed magnetic phase already in zero applied magnetic field. We note that this does not prohibit the existence of a superconducting phase with similarly high $T_{c}$ compared to samples with magnetic phases of different magnitudes.

\section{CONCLUSION}

We have presented an extensive neutron scattering study which characterizes the magnetic order and fluctuations in the stage- 4 oxygen doped $\mathrm{La}_{2} \mathrm{CuO}_{4+\delta}$ superconductor.

The lack of a spin gap in the low-energy magnetic spectrum is interpreted as an indication that superconductivity and spin fluctuations coexist microscopically in an intertwined phase with no sign of competition. Notably this happens in a single-layer cuprate superconductor that exhibits a clean superconducting transition with one of the highest critical temperatures of $T_{c}=42 \mathrm{~K}$.

On the other hand, we consider magnetic order to be a separate electronic phase competing with superconductivity, in agreement with previous interpretations from the literature [7]. The lack of a magnetic field effect on the elastic stripe signal, shows that our sample contains a fully developed magnetic phase. This observation contrasts previous reports of a magnetic field enhancement $[50,55,69]$, and is possibly explained by discrepancies in the initial magnetic volume fraction of crystals which have been doped under different oxygenation procedures.

The $x y z$ polarization analysis revealed the presence of equal fractions of spin stripe modulations oriented along either of the tetragonal $a_{T}$ axis or $b_{T}$ axis. Corroborated with a study previously published by some of us [65], our data supports the hypothesis that the magnetic fluctuations in LCOO are likely isotropic in nature, but further work is needed to confirm this.

In conclusion, we propose a picture of electronic phase separation in LCOO samples into two competing phases, where one exhibits magnetic stripe order while the other hosts superconductivity intertwined with low-energy spin fluctuations.

\section{ACKNOWLEDGMENTS}

We give a special thanks to Niels Hessel Andersen for his work within this field of science throughout his long career, and particularly thank him for his contributions to this project. He will be missed, both as a colleague and as a friend. We are grateful for the access to neutron beamtime at the ILL and HZB neutron facilities. This project was supported by the Danish Council for Independent Research through DANSCATT. A.T.R. acknowledges support from the Carlsberg Foundation. H.J. acknowledges funding from the EU Horizon 2020 program under the Marie Sklodowska-Curie Grant No.
701647. A.-E.T. was supported through the ILL Ph.D. program. S.H.D. acknowledges support from NordForsk through NNSP, Project No. 87865. B.O.W. was supported for sample production and data interpretation by the US Department of Energy Basic Energy Sciences under Contract No. DE-FG0200ER45801.

\section{APPENDIX A: MAGNETIC SUSCEPTIBILITY}

In order to accurately determine the magnetic (volume) susceptibility of the sample in SI units, from the magnetic moment measurements, the following formula was applied:

$$
\chi=4 \pi \frac{\mu}{V H_{\text {internal }}},
$$

where $\mu$ is the measured magnetic moment in electromagnetic units (emu), $V$ is the sample's volume in $\mathrm{cm}^{3}$, and $H_{\text {internal }}$ is the strength of the internal magnetic field. It should be noted that the internal magnetic field differs from the applied one $\left(H_{\text {applied }}\right)$ by a demagnetizing factor $(N)$ :

$$
H_{\text {internal }}=\frac{1}{1-N} H_{\text {applied }}
$$

which is valid only in the Meissner state, i.e., for very small applied magnetic fields. Our measurements were performed under $H_{\text {applied }}=6.4 \mathrm{mT}$.

If we treat our cubic samples as spheres, in which case the demagnetizing factor has the value $N=1 / 3$, we obtain:

$$
H_{\text {internal }}=1.5 \times H_{\text {applied }},
$$

For an accurate measure of the sample's volume, the density $(\rho)$ of LCOO is used in the calculations as follows:

$$
\rho=\frac{m_{\text {UnitCell }}}{V_{\text {UnitCell }}} .
$$

where $V_{\text {UnitCell }}$ is the unit cell volume calculated with the lattice parameters taken from Ref. [83] for oxygen doping $\delta=0.08$ at a temperature of $10 \mathrm{~K}$ :

$$
\begin{aligned}
V_{\text {UnitCell }} & =a \times b \times c=5.33 \AA \times 5.39 \AA \times 13.16 \AA \\
& =378.07 \AA^{3}=378.07 \times 10^{-24} \mathrm{~cm}^{3} .
\end{aligned}
$$

The number of atoms per unit cell and the atomic masses of the three components of LCOO (shown in Table III) are used to determine the mass of the unit cell:

$$
\begin{aligned}
m_{\text {UnitCell }} & =m_{\mathrm{La}} n_{\mathrm{La}}+m_{\mathrm{Sr}} n_{\mathrm{Sr}}+m_{\mathrm{Cu}} n_{\mathrm{Cu}}+m_{O} n_{O} \\
& =2.70 \times 10^{-21} \mathrm{~g} .
\end{aligned}
$$

TABLE III. The mass of one atom was calculated as the ratio between the atomic mass and Avogadro's number. The number of atoms is presented for a conventional unit cell of $\mathrm{LCO}+\mathrm{O}$ with oxygen doping $\delta=0.08$.

\begin{tabular}{lcc}
\hline \hline Element & Atom's mass $(\mathrm{m})(\mathrm{g})$ & No. of atoms/unit cell $(\mathrm{n})$ \\
\hline $\mathrm{La}$ & $2.31 \times 10^{-22}$ & 8 \\
$\mathrm{Cu}$ & $1.05 \times 10^{-22}$ & 4 \\
$\mathrm{O}$ & $2.66 \times 10^{-23}$ & 16.32 \\
\hline
\end{tabular}


Finally, following Eq. (A4), the density of LCOO with doping $x=0.08$ is $\boldsymbol{\rho}=\mathbf{7 . 1 4} \mathbf{g} / \mathbf{c m}^{3}$. This value is afterwards used as the division factor of the mass in order to obtain an accurate estimation of the sample's volume.

The calculated susceptibility is then plotted against the temperature as it can be observed in Fig. 1 in the main text. In an ideal case, the graph will have the shape of a sigmoid function constrained in between 0 (corresponding to the antiferromagnetic state above $T_{c}$ ) and -1 (corresponding to the perfect diamagnetic response of the material below $T_{c}$ ).

\section{APPENDIX B: SUPERCONDUCTING CRITICAL TEMPERATURE MEASURED BY POLARIZED NEUTRON SCATTERING}

Magnetic susceptibility measurements are traditionally used in determining the critical temperature, and implicitly the doping level, of superconductors due to their high accuracy. However, the fact that only small crystallites are used makes them unreliable in evaluating the properties of the entire bulk of the sample. Alternatively, one can use polarised neutron imaging or tomography to accurately evaluate doping distributions throughout large single crystals [84]. Here we propose yet another method to determine the superconducting critical temperature by following, as a function of increased temperature, the depolarization of the neutron beam induced by the trapped magnetic flux inside the sample.

The experiment, which we performed on Thales at the ILL, starts with trapping a magnetic flux in form of vortex lines inside the superconducting sample by slowly cooling $(1 \mathrm{~K} / \mathrm{min})$ it in a small applied vertical magnetic field ( $\| c$ axis). At base temperature, the magnetic field which will guide the neutron polarization in the following, is switched as to point along the in-plane $x$ direction. We have then measured the intensity of the $(2,0,0)$ Bragg peak which is purely nuclear and should therefore not contribute to the spin flip channel. However, significant intensity appears as a consequence of beam depolarization, which is caused by the nonadiabatic field change which the neutron undergoes when crossing the region of trapped magnetic flux in the sample. This depolarization effect disappears sharply at $T=T c$ when the samples exits the superconducting state and the outer magnetic guide field penetrates the sample homogeneously; the observed intensity then drops to a constant low value which reflects the finite natural beam polarization.

The measurement is depicted in Fig. 6 and shows a fairly sharp transition at $\sim 40 \mathrm{~K}$, in agreement with the critical temperature we have extracted from magnetic susceptibility data. We attribute the peculiar slight increase in intensity at around $\sim 28 \mathrm{~K}$ to a phase transition from a vortex lattice to a vortex liquid phase [85].

We are thus confident that the magnetic susceptibility measurements we have presented in Fig. 1(a) are representative for the majority of our $\mathrm{LCO}+\mathrm{O}$ sample.

\section{APPENDIX C: ABSOLUTE NORMALIZATION OF MAGNETIC CROSS SECTION}

The normalization process of the magnetic cross section follows closely the procedure explained by $\mathrm{Xu}$ et al. in

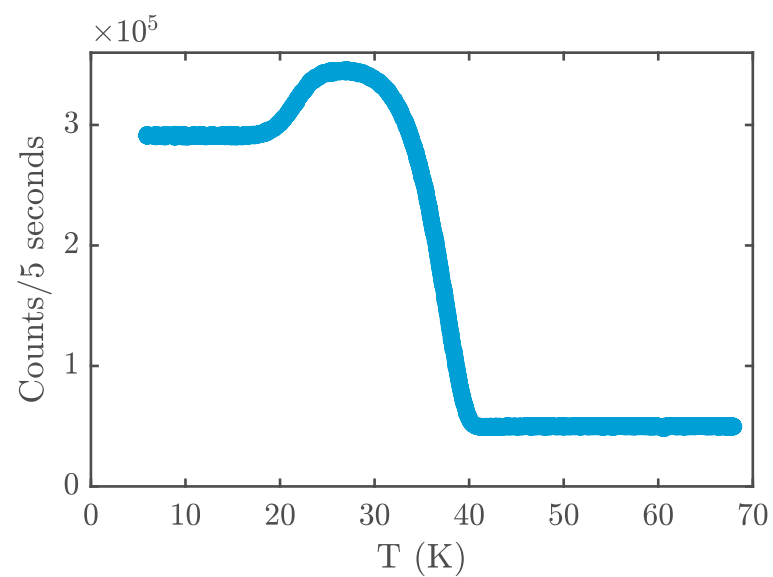

FIG. 6. Measured intensity of the spin flip channel at $(2,0,0)$ Bragg position as a function of temperature.

Ref. [71]. Here we exemplify how we have applied it on our data, in particular the data collected on IN12. We note that special attention should be paid to the units used.

The dynamic susceptibility is defined as:

$$
\chi^{\prime \prime}(\mathbf{Q}, \omega)=\frac{\pi}{2} \mu_{B}^{2}\left(1-e^{-\hbar \omega / k_{B} T}\right) \frac{13.77\left(b^{-1}\right) \tilde{I}(\mathbf{Q}, E)}{|f(\mathbf{Q})|^{2} e^{-2 W} N k_{f} R_{0}} .
$$

We will now illustrate the calculation of each of the terms for the IN12 data.

(i) $N k_{f} R_{0}$ : the resolution volume

The resolution volume is obtained from the sample phonon scattering measured as a constant energy scan:

$$
N k_{f} R_{0}=\frac{\int \tilde{I}(\mathbf{Q}, E) d \mathbf{q}}{e^{-2 W}\left|F_{N}(\mathbf{G})\right|^{2} \cos ^{2}(\beta) \frac{m}{M} \frac{(\hbar \mathbf{Q})^{2}}{2 m} \frac{n}{\hbar \omega} \frac{1}{d \omega / d q}},
$$

where $\int \tilde{I}(\mathbf{Q}, E) d \mathbf{q}$ is the average integrated intensity of the two phonon branches. $e^{-2 W}$ is the Debye-Waller factor and $\beta$ is the angle between $\mathbf{Q}$ and the polarization of the phonon. Both $e^{-2 W}$ and $\cos ^{2}(\beta)$ are assumed 1. $F_{N}(\mathbf{G})$ is the structure factor of the phonon, it can easily be obtained from VESTA [86] and should be used in units of barns; $m$ is the neutron mass and $M$ is the is the mass of all the atoms in the unit cell (see Table III); $n=1 /\left(1-e^{-E / k_{B} T}\right.$ ) is the Bose factor of the phonon, where $E$ is the energy at which the phonon was measured, $k_{B}$ is the Boltzmann constant, and $T$ is the temperature at which the phonon was measured; $d \omega / d q$ is the phonon velocity where $\omega$ is the energy at which the phonon was measured and $q$ is obtained as half the distance between the two phonon branches in reciprocal lattice units. The term $\frac{(\hbar \mathbf{Q})^{2}}{2 m}$ should be calculated in units of $\mathrm{meV}$.

In the case of our acoustic phonon measured on IN12 at $(2,0,0), 3 \mathrm{meV}$ and $280 \mathrm{~K}$, we obtain the numerical values shown in Table IV.

(ii) $f(\mathbf{Q})$ : the form factor

In the case of $\mathrm{LCOO}$ it is sufficient to use the $\mathrm{Cu}$ form factor since it is the only atom that is responsible for the magnetic scattering

$$
f(\mathbf{Q})=A e^{-a\left(\frac{\mathbf{Q}}{4 \pi}\right)^{2}}+B e^{-b\left(\frac{\mathbf{Q}}{4 \pi}\right)^{2}}+C e^{-c\left(\frac{\mathbf{Q}}{4 \pi}\right)^{2}}+D,
$$


TABLE IV. Parameters corresponding to the acoustic phonon measured on IN12.

\begin{tabular}{lcccccc}
\hline \hline Term & $F_{N}(\mathbf{G})$ & $m / M$ & $\frac{(\hbar \mathbf{Q})^{2}}{2 m}$ & $\frac{n_{q}}{\hbar \omega}$ & $d \omega / d q$ & $\begin{array}{c}N k_{f} R_{0} \\
\text { Unit }\end{array}$ \\
barns & - & $\mathrm{meV}$ & $\mathrm{meV}^{-1}$ & $\mathrm{meV} / \mathrm{r} .1 . \mathrm{u}$. & $\mathrm{meV} / \mathrm{barns}$ \\
Value & 91 & $\frac{1}{1617}$ & 11.57 & 2.85 & 35.76 & 0.0025 \\
\hline \hline
\end{tabular}

where the coefficients $A, a, B, b, C, c$, and $D$ can easily be found tabulated [87] for $\mathrm{Cu}^{2+}$.

The other remaining factors in Eq. (C1) are the Bose factor of the measured magnetic signal $1 /\left(1-e^{-E / k_{B} T}\right)$, the DebyeWaller factor $e^{-2 W}$ assumed 1, the Bohr magneton $\mu_{B}$ and the amplitude of one of the incommensurate magnetic peaks $\tilde{I}(\mathbf{Q}, E)$.

Furthermore, in order to obtain the Q-integrated local susceptibility $\left(\chi^{\prime \prime}(\omega)\right)$, as presented in Ref. [45] for an underdoped LBCO sample, an additional integration over all the peaks in the Brillouin zone (BZ) is needed:

$$
\chi^{\prime \prime}(\omega)=\frac{2 \pi \sigma_{h} \sigma_{k} n_{\text {peaks }}}{a^{*} b^{*}} \chi^{\prime \prime}(\mathbf{Q}, \omega),
$$

where $\sigma_{h}$ and $\sigma_{k}$ are the widths of the Gaussian fits to the peaks in $h$ - and $k$-directions, $n_{\text {peaks }}$ is the number of peaks in the $\mathrm{BZ}$ and $a^{*}, b^{*}$ are the reciprocal lattice parameters. In our orthorhombic notation there are two peaks in the BZ. For these measurements we have only performed scans along one of the in-plane reciprocal space directions, and will thus need to estimate $\sigma_{h} \sigma_{k}$. A naive estimate would be to assume $\sigma_{h}=\sigma_{k}$. However, in Ref. [65] we measured a coarse grid on the same sample, and found the peak to be wider in the longitudinal direction. We fitted the peak using a two-dimensional Gaussian, and found that $\sigma_{h} \sigma_{k} \approx 3.0 \sigma_{k}^{2}$. These values have then been used in Eq. (C4).

$\chi^{\prime \prime}(\omega)$ does not depend on the choice of unit cell (tetragonal or orthorhombic), meaning that all variations of the parameters (such as the number of $\mathrm{Cu}$ per unit cell or the lattice parameters) eventually cancel out. We can thus make a direct comparison, as shown in Fig. 7, between the magnetic spectral weight of our sample and the one of an underdoped LBCO crystal presented in the literature. We note that there is a rather large uncertainty in the normalization procedure, as

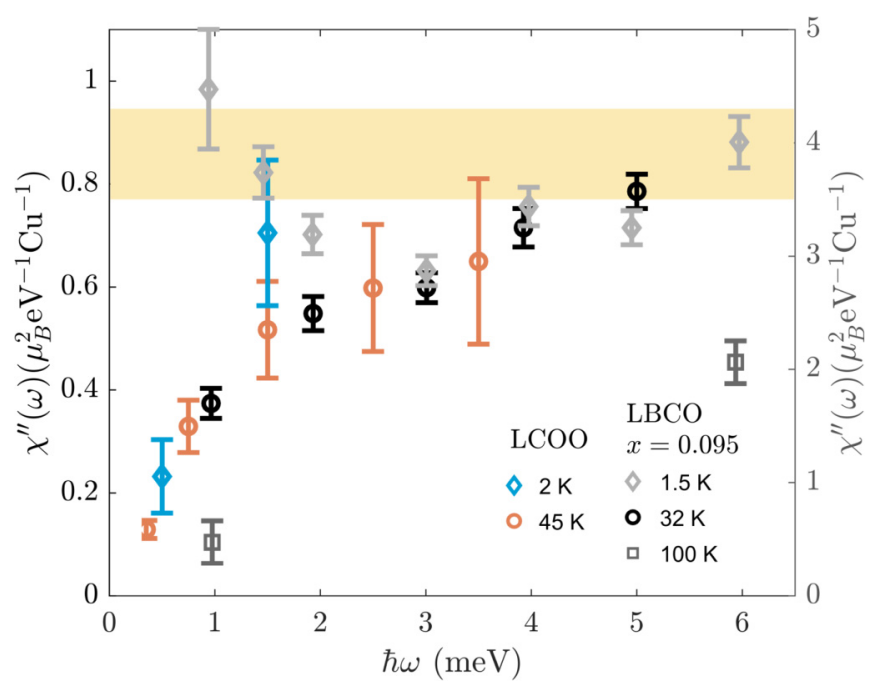

FIG. 7. Dynamic susceptibility $\chi^{\prime \prime}(\omega)$ measured at temperatures inside $(2 \mathrm{~K})$ and outside $(45 \mathrm{~K})$ the superconducting dome. All data has been measured at Thales (Th1) with constant $k_{f}=1.55 \AA^{-1}$. In gray and black symbols, we show the magnetic susceptibility measured inside and outside the superconducting state by $\mathrm{Xu}$ et al. [45] on an underdoped $x=0.095 \mathrm{LSBCO}$ sample with $T_{c}=32 \mathrm{~K}$. The shaded area indicates the magnitude of the spin waves in the parent compound LCO, as presented by Xu et al. [45], and is connected to the right-hand side axis.

we make multiple assumptions about the shape of the signal. In addition, our scan might not pass through the top of the peak; this can easily lead to an observed intensity which is $\sim 20 \%$ smaller than the actual intensity.

Despite these shortcomings of the absolute normalization process, the procedure allows for a rough comparison of the magnetic spectral weight of the very low-energy fluctuations in LCOO with that of underdoped LBCO and the parent compound LCO [45]. As shown in Fig. 7, we find that the magnetic weight in LCOO is five times lower than in LBCO and undoped LCO. We ascribe this to the fact that some regions of our LCOO crystal show AFM order, which we do not include in the analysis.
[1] M. Vojta, Lattice symmetry breaking in cuprate superconductors: Stripes, nematics, and superconductivity, Adv. Phys. 58, 699 (2009).

[2] J. M. Tranquada, Spins, stripes, and superconductivity in holedoped cuprates, in AIP Conference Proceedings (AIP, Melville, NY, 2013), Vol. 1550, pp. 114-187.

[3] B. Keimer, S. A. Kivelson, M. R. Norman, S. Uchida, and J. Zaanen, From quantum matter to high-temperature superconductivity in copper oxides, Nature 518, 179 (2015).

[4] E. Fradkin, S. A. Kivelson, and J. M. Tranquada, Colloquium: Theory of intertwined orders in high temperature superconductors, Rev. Mod. Phys. 87, 457 (2015).

[5] D. F. Agterberg, J. S. Davis, S. D. Edkins, E. Fradkin, H. D. J. Va, S. A. Kivelson, P. A. Lee, L. Radzihovsky, J. M. Tranquada, and Y. Wang, The physics of pair-density waves: Cuprate superconductors and beyond, Annu. Rev. Condens. Matter Phys. 11, 231 (2020).

[6] D. J. Scalapino, A common thread: The pairing interaction for unconventional superconductors, Rev. Mod. Phys. 84, 1383 (2012).

[7] M. Julien, Magnetic order and superconductivity in $\mathrm{La}_{2-x} \mathrm{Sr}_{x} \mathrm{CuO}_{4}$ : A review, Phys. B: Condens. Matter 329, 693 (2003).

[8] M. Fujita, H. Hiraka, M. Matsuda, M. Matsuura, T. J. $\mathrm{M}$, S. Wakimoto, G. $\mathrm{Xu}$, and $\mathrm{K}$. Yamada, Progress in neutron scattering studies of spin excitations in high- $\mathrm{T}_{c}$ cuprates, J. Phys. Soc. Jpn. 81, 011007 (2012). 
[9] M. Hücker, Structural aspects of materials with static stripe order, Physica C 481, 3 (2012).

[10] B. O. Wells, Y. S. Lee, M. A. Kastner, R. J. Christianson, R. J. Birgeneau, K. Yamada, Y. Endoh, and G. Shirane, Incommensurate spin fluctuations in high-transition temperature superconductors, Science 277, 1067 (1997).

[11] M. Kofu, S. H. Lee, M. Fujita, H. J. Kang, H. Eisaki, and K. Yamada, Hidden Quantum Spin-Gap State in the Static Stripe Phase of High-Temperature $\mathrm{La}_{2-x} \mathrm{Sr}_{x} \mathrm{CuO}_{4}$ Superconductors, Phys. Rev. Lett. 102, 047001 (2009).

[12] K. Yamada, C. H. Lee, K. Kurahashi, J. Wada, S. Wakimoto, S. Ueki, H. Kimura, Y. Endoh, S. Hosoya, G. Shirane, R. J. Birgeneau, M. Greven, M. A. Kastner, and Y. J. Kim, Doping dependence of the spatially modulated dynamical spin correlations and the superconducting-transition temperature in $\mathrm{La}_{2-x} \mathrm{Sr}_{x} \mathrm{CuO}_{4}$, Phys. Rev. B 57, 6165 (1998).

[13] Y. S. Lee, R. J. Birgeneau, M. A. Kastner, Y. Endoh, S. Wakimoto, K. Yamada, R. W. Erwin, S.-H. Lee, and G. Shirane, Neutron-scattering study of spin-density wave order in the superconducting state of excess-oxygen-doped $\mathrm{La}_{2} \mathrm{CuO}_{4+y}$, Phys. Rev. B 60, 3643 (1999).

[14] H. Kimura, H. Matsushita, K. Hirota, Y. Endoh, K. Yamada, G. Shirane, Y. S. Lee, M. A. Kastner, and R. J. Birgeneau, Incommensurate geometry of the elastic magnetic peaks in superconducting $\mathrm{La}_{1.88} \mathrm{Sr}_{0.12} \mathrm{CuO}_{4}$, Phys. Rev. B 61, 14366 (2000).

[15] J. M. Tranquada, B. J. Sternlieb, J. D. Axe, Y. Nakamura, and S. Uchida, Evidence for stripe correlations of spins and holes in copper oxide superconductors, Nature 375, 561 (1995).

[16] M. Fujita, H. Goka, K. Yamada, J. M. Tranquada, and L. P. Regnault, Stripe order, depinning, and fluctuations in $\mathrm{La}_{1.875} \mathrm{Ba}_{0.125} \mathrm{CuO}_{4}$ and $\mathrm{La}_{1.875} \mathrm{Ba}_{0.075} \mathrm{Sr}_{0.050} \mathrm{CuO}_{4}$, Phys. Rev. B 70, 104517 (2004).

[17] M. Hucker, Z. M. v, G. D. Gu, Z. J. Xu, J. S. Wen, G. Xu, H. J. Kang, A. Zheludev, and J. M. Tranquada, Stripe order in superconducting $\mathrm{La}_{2-x} \mathrm{Ba}_{x} \mathrm{CuO}_{4}(0.095 \leqslant x \leqslant 0.155)$, Phys. Rev. B 83, 104506 (2011).

[18] T. Suzuki, T. Goto, K. Chiba, T. Shinoda, T. Fukase, H. Kimura, K. Yamada, M. Ohashi, and Y. Yamaguchi, Observation of modulated magnetic long-range order in $\mathrm{La}_{1.88} \mathrm{Sr}_{0.12} \mathrm{CuO}_{4}$, Phys. Rev. B 57, R3229 (1998).

[19] S. Katano, M. Sato, K. Yamada, T. Suzuki, and T. Fukase, Enhancement of static antiferromagnetic correlations by magnetic field in a superconductor $\mathrm{La}_{2-x} \mathrm{Sr}_{x} \mathrm{CuO}_{4}$ with $x=0.12$, Phys. Rev. B 62, R14677 (2000).

[20] B. Lake, H. M. Rønnow, N. B. Christensen, G. Aeppli, K. Lefmann, D. F. McMorrow, P. Vorderwisch, P. Smeibidl, N. Mangkorntong, T. Sasagawa, M. Nohara, H. Takagi, and T. E. Mason, Antiferromagnetic order induced by an applied magnetic field in a high-temperature superconductor, Nature $\mathbf{4 1 5}$, 299 (2002).

[21] J. Chang, N. B. Christensen, C. Niedermayer, K. Lefmann, H. M. Rønnow, D. F. McMorrow, A. Schneidewind, P. Link, A. Hiess, M. Boehm, R. Mottl, S. Pailhés, N. Momono, M. Oda, M. Ido, and J. Mesot, Magnetic-Field-Induced Soft-Mode Quantum Phase Transition in the High-Temperature Superconductor $\mathrm{La}_{1.855} \mathrm{Sr}_{0.145} \mathrm{CuO}_{4}$ : An Inelastic Neutron-Scattering Study, Phys. Rev. Lett. 102, 177006 (2009).

[22] B. M. Andersen, M. Schmid, S. Graser, P. J. Hirschfeld, and A. P. Kampf, Effects of impurities and vortices on the low- energy spin excitations in high- $T_{c}$ materials, J. Phys. Chem. Solids 72, 358 (2011).

[23] M. Hücker, M. v. Zimmermann, Z. J. Xu, J. S. Wen, G. D. $\mathrm{Gu}$, W. Tian, J. Zarestky, and J. M. Tranquada, Zn-doping dependence of stripe order in $\mathrm{La}_{1.905} \mathrm{Ba}_{0.095} \mathrm{CuO}_{4}$, J. Supercond. Nov. Magn. 24, 1229 (2011).

[24] J. Wen, Q. Jie, Q. Li, M. Hücker, M. v. Zimmermann, S. J. Han, Z. Xu, D. K. Singh, R. M. Konik, L. Zhang et al., Uniaxial linear resistivity of superconducting $\mathrm{La}_{1.905} \mathrm{Ba}_{0.095} \mathrm{CuO}_{4}$ induced by an external magnetic field, Phys. Rev. B 85, 134513 (2012).

[25] J. Wen, Z. Xu, G. Xu, Q. Jie, M. Hücker, A. Zheludev, W. Tian, B. L. Winn, J. L. Zarestky, D. K. Singh et al., Probing the connections between superconductivity, stripe order, and structure in $\mathrm{La}_{1.905} \mathrm{Ba}_{0.095} \mathrm{Cu}_{1-y} \mathrm{Zn}_{y} \mathrm{O}_{4}$, Phys. Rev. B 85, 134512 (2012).

[26] M. Frachet, I. Vinograd, R. Zhou, S. Benhabib, S. Wu, H. Mayaffre, S. Krämer, S. K. Ramakrishna, A. P. Reyes, J. Debray, T. Kurosawa, N. Momono, M. Oda, S. Komiya, S. Ono, M. Horio, J. Chang, C. Proust, D. LeBoeuf, and M.-H. Julien, Hidden magnetism at the pseudogap critical point of a cuprate superconductor, Nat. Phys. 16, 1064 (2020).

[27] Z. Guguchia, D. Das, C. N. Wang, T. Adachi, N. Kitajima, M. Elender, F. Brückner, S. Ghosh, V. Grinenko, T. Shiroka, M. Müller, C. Mudry, C. Baines, M. Bartkowiak, Y. Koike, A. Amato, J. M. Tranquada, H.-H. Klauss, C. W. Hicks, and H. Luetkens, Using Uniaxial Stress to Probe the Relationship between Competing Superconducting States in a Cuprate with Spin-Stripe Order, Phys. Rev. Lett. 125, 097005 (2020).

[28] B. Lake, G. Aeppli, T. Mason, A. Schröder, D. McMorrow, K. Lefmann, M. Isshiki, M. Nohara, H. Takagi, and S. Hayden, Spin gap and magnetic coherence in a clean high-temperature superconductor, Nature 400, 43 (1999).

[29] K. Yamada, S. Wakimoto, G. Shirane, C. H. Lee, M. A. Kastner, S. Hosoya, M. Greven, Y. Endoh, and R. J. Birgeneau, Direct Observation of a Magnetic Gap in Superconducting $\mathrm{La}_{1.85} \mathrm{Sr}_{0.15} \mathrm{CuO}_{4}\left(\mathrm{~T}_{c}=37.3 \mathrm{~K}\right)$, Phys. Rev. Lett. 75, 1626 (1995).

[30] J. Chang, A. P. Schnyder, R. Gilardi, H. M. Rønnow, S. Pailhes, N. B. Christensen, C. Niedermayer, D. F. McMorrow, A. Hiess, A. Stunault, M. Enderle, B. Lake, O. Sobolov, N. Momono, M. Oda, M. Ido, C. Mudry, and J. Mesot, Magnetic-Field-Induced Spin Excitations and Renormalized Spin Gap of the Underdoped $\mathrm{La}_{1.895} \mathrm{Sr}_{0.105} \mathrm{CuO}_{4}$ Superconductor, Phys. Rev. Lett. 98, 077004 (2007).

[31] H. Hiraka, Y. Endoh, M. Fujita, Y. S. Lee, J. Kulda, A. Ivanov, and R. J. Birgeneau, Spin fluctuations in the underdoped high- $\mathrm{T}_{c}$ cuprate $\mathrm{La}_{1.93} \mathrm{Sr}_{0.07} \mathrm{CuO}_{4}$, J. Phys. Soc. Jpn. 70, 853 (2001).

[32] H. Jacobsen, I. A. Zaliznyak, A. T. Savici, B. L. Winn, S. Chang, M. Hücker, G. D. Gu, and J. M. Tranquada, Neutron scattering study of spin ordering and stripe pinning in superconducting $\mathrm{La}_{1.93} \mathrm{Sr}_{0.07} \mathrm{CuO}_{4}$, Phys. Rev. B 92, 174525 (2015).

[33] B. Lake, G. Aeppli, K. N. Clausen, D. F. McMorrow, K. Lefmann, N. E. Hussey, N. Mangkorntong, M. Nohara, H. Takagi, T. E. Mason, and A. Schröder, Spins in the vortices of a high-temperature superconductor, Science 291, 1759 (2001).

[34] J. M. Tranquada, C. H. Lee, K. Yamada, Y. S. Lee, L. P. Regnault, and H. M. Rønnow, Evidence for an incommensurate magnetic resonance in $\mathrm{La}_{2-x} \mathrm{Sr}_{x} \mathrm{CuO}_{4}$, Phys. Rev. B 69, 174507 (2004). 
[35] J. M. Tranquada, G. D. Gu, M. Hücker, Q. Jie, H.. Kang, R. Klingeler, Q. Li, N. Tristan, J. S. Wen, G. Y. Xu, Z. J. $\mathrm{Xu}$, J. Zhou, and M. v. Zimmermann, Evidence for unusual superconducting correlations coexisting with stripe order in $\mathrm{La}_{1.875} \mathrm{Ba}_{0.125} \mathrm{CuO}_{4}$, Phys. Rev. B 78, 174529 (2008).

[36] A. T. Savici, A. Fukaya, I. M. Gat-Malureanu, T. Ito, P. L. Russo, Y. J. Uemura, C. R. Wiebe, P. P. Kyriakou, G. J. MacDougall, M. T. Rovers, G. M. Luke, K. M. Kojima, M. Goto, S. Uchida, R. Kadono, K. Yamada, S. Tajima, T. Masui, H. Eisaki, N. Kaneko, M. Greven, and G. D. Gu, Muon Spin Relaxation Studies of Magnetic-Field-Induced Effects in High$T_{c}$ Superconductors, Phys. Rev. Lett. 95, 157001 (2005).

[37] Q. Li, M. Hucker, G. D. Gu, A. M. Tsvelik, and J. M. Tranquada, Two-Dimensional Superconducting Fluctuations in Stripe-Ordered $\mathrm{La}_{1.875} \mathrm{Ba}_{0.125} \mathrm{CuO}_{4}$, Phys. Rev. Lett. 99, 067001 (2007)

[38] J. Arai, T. Ishiguro, T. Goko, S. Iigaya, K. Nishiyama, I. Watanabe, and K. Nagamine, $\mu$ sr study of magnetic order in lto and ltt phase of $\mathrm{La}_{2-x} M_{x} \mathrm{Cu}_{1-y} \mathrm{Zn}_{y} \mathrm{O}_{4}(M=\mathrm{Sr}$, Ba), J. Low Temp. Phys. 131, 375 (2003).

[39] N. Christensen, J. Chang, J. Larsen, M. Fujita, M. Oda, M. Ido, N. Momono, E. Forgan, A. Holmes, J. Mesot et al., Bulk charge stripe order competing with superconductivity in $\mathrm{La}_{2-x} \mathrm{Sr}_{x} \mathrm{CuO}_{4}$ $(x=0.12)$, arXiv:1404.3192.

[40] T. P. Croft, C. Lester, M. S. Senn, A. Bombardi, and S. M. Hayden, Charge density wave fluctuations in $\mathrm{La}_{2-x} \mathrm{Sr}_{x} \mathrm{CuO}_{4}$ and their competition with superconductivity, Phys. Rev. B 89, 224513 (2014).

[41] V. Thampy, M. P. M. Dean, N. B. Christensen, L. Steinke, Z. Islam, M. Oda, M. Ido, N. Momono, S. B. Wilkins, and J. P. Hill, Rotated stripe order and its competition with superconductivity in $\mathrm{La}_{1.88} \mathrm{Sr}_{0.12} \mathrm{CuO}_{4}$, Phys. Rev. B 90, 100510(R) (2014).

[42] Z. Zhang, R. Sutarto, F. He, F. C. Chou, L. Udby, S. L. Holm, Z. H. Zhu, W. A. Hines, J. I. Budnick, and B. O. Wells, Nematicity and Charge Order in Superoxygenated $\mathrm{La}_{2-x} \mathrm{Sr}_{x} \mathrm{CuO}_{4+y}$, Phys. Rev. Lett. 121, 067602 (2018).

[43] A. T. Rømer, J. Chang, N. B. Christensen, B. M. Andersen, K. Lefmann, L. Mähler, J. Gavilano, R. Gilardi, C. Niedermayer, H. M. Rønnow, A. Schneidewind, P. Link, M. Oda, M. Ido, N. Momono, and J. Mesot, Glassy low-energy spin fluctuations and anisotropy gap in $\mathrm{La}_{1.88} \mathrm{Sr}_{0.12} \mathrm{CuO}_{4}$, Phys. Rev. B 87, 144513 (2013).

[44] J. Wen, Z. Xu, G. Xu, J. M. Tranquada, G. Gu, S. Chang, and H. J. Kang, Magnetic field induced enhancement of spin-order peak intensity in $\mathrm{La}_{1.875} \mathrm{Ba}_{0.125} \mathrm{CuO}_{4}$, Phys. Rev. B 78, 212506 (2008)

[45] Z. Xu, C. Stock, S. Chi, A. I. Kolesnikov, G. Xu, G. Gu, and J. M. Tranquada, Neutron-Scattering Evidence for a Periodically Modulated Superconducting Phase in the Underdoped Cuprate $\mathrm{La}_{1.905} \mathrm{Ba}_{0.095} \mathrm{CuO}_{4}$, Phys. Rev. Lett. 113, 177002 (2014)

[46] J. Tranquada, H. Woo, T. Perring, H. Goka, G. Gu, G. Xu, M. Fujita, and K. Yamada, Quantum magnetic excitations from stripes in copper oxide superconductors, Nature 429, 534 (2004)

[47] N. B. Christensen, D. F. McMorrow, H. M. Rønnow, B. Lake, S. M. Hayden, G. Aeppli, T. G. Perring, M. Mangkorntong, M. Nohara, and H. Tagaki, Dispersive Excitations in the HighTemperature Superconductor $\mathrm{La}_{2-x} \mathrm{Sr}_{x} \mathrm{CuO}_{4}$, Phys. Rev. Lett. 93, 147002 (2004).
[48] H. E. Mohottala, B. O. Wells, J. I. Budnick, W. A. Hines, C. Niedermayer, L. Udby, C. Bernhard, A. R. Moodenbaugh, and F. Chou, Phase separation in superoxygenated $\mathrm{La}_{2-x} \mathrm{Sr}_{x} \mathrm{CuO}_{4+y}$. Nat. Mater. 5, 377 (2006).

[49] A. T. Savici, Y. Fudamoto, I. M. Gat, T. Ito, M. I. Larkin, Y. J. Uemura, G. M. Luke, K. M. Kojima, Y. S. Lee, M. A. Kastner, R. J. Birgeneau, and K. Yamada, Muon spin relaxation studies of incommensurate magnetism and superconductivity in stage$4 \mathrm{La}_{2} \mathrm{CuO}_{4.11}$ and $\mathrm{La}_{1.88} \mathrm{Sr}_{0.12} \mathrm{CuO}_{4}$, Phys. Rev. B 66, 014524 (2002).

[50] B. Khaykovich, Y. S. Lee, R. Erwin, S. H. Lee, S. Wakimoto, K. J. Thomas, M. A. Kastner, and R. J. Birgeneau, Enhancement of long-range magnetic order by magnetic field in superconducting $\mathrm{La}_{2} \mathrm{CuO}_{4+y}$, Phys. Rev. B 66, 014528 (2002).

[51] D. Vaknin, S. K. Sinha, D. E. Moncton, D. C. Johnston, J. M. Newsam, C. R. Safinya, and H. E. King, Antiferromagnetism in $\mathrm{La}_{2} \mathrm{Cu}_{4-y}$, Phys. Rev. Lett. 58, 2802 (1987).

[52] H. E. Mohottala, B. O. Wells, J. I. Budnick, W. A. Hines, C Niedermayer, and F. C. Chou, Flux pinning and phase separation in oxygen-rich $\mathrm{La}_{2-x} \mathrm{Sr}_{x} \mathrm{CuO}_{4+y}$, Phys. Rev. B 78, 064504 (2008).

[53] N. Poccia, A. Ricci, G. Campi, M. Fratini, A. Puri, D. Di Gioacchino, A. Marcelli, M. Reynolds, M. Burghammer, N. L. Saini, G. Aeppli, and A. Bianconi, Optimum inhomogeneity of local lattice distortions in $\mathrm{La}_{2} \mathrm{CuO}_{4+y}$, Proc. Natl. Acad. Sci. USA 109, 15685 (2012).

[54] N. Poccia, A. Ricci, G. Campi, and A. Bianconi, Dislocations as a boundary between charge density wave and oxygen rich phases in a cuprate high temperature superconductor, Supercond. Sci. Technol. 30, 035016 (2017).

[55] B. Khaykovich, R. J. Birgeneau, F. C. Chou, R. W. Erwin, M. A. Kastner, S. H. Lee, Y. S. Lee, P. Smeibidl, P. Vorderwisch, and S. Wakimoto, Effect of a magnetic field on long-range magnetic order in stage- 4 and stage- 6 superconducting $\mathrm{La}_{2} \mathrm{CuO}_{4+y}$, Phys. Rev. B 67, 054501 (2003).

[56] S. R. Dunsiger, Y. Zhao, Z. Yamani, W. J. L. Buyers, H. Dabkowska, and B. D. Gaulin, Incommensurate spin ordering and fluctuations in underdoped $\mathrm{La}_{2-x} \mathrm{Ba}_{x} \mathrm{CuO}_{4}$, Phys. Rev. B 77, 224410 (2008).

[57] H. L. Bhat, Introduction to Crystal Growth (CRC Press, Boca Raton, FL, 2015).

[58] K. Habicht, D. L. Quintero-Castro, R. Toft-Petersen, M. Kure, L. Mäde, F. Groitl, and M. D. Le, The upgraded cold neutron triple-axis spectrometer FLEXX-enhanced capabilities by new instrumental options, in EPJ Web of Conferences (EDP Sciences, Les Ulis, France, 2015), Vol. 83, p. 03007.

[59] M. D. Le, L. Q. D, R. Toft-Petersen, F. Groitl, M. Skoulatos, K. C. Rule, and K. Habicht, Gains from the upgrade of the cold neutron triple-axis spectrometeer FLEXX at the BER-II reactor, Nucl. Instrum. Methods A 729, 220 (2013).

[60] K. Schmalzl, W. Schmidt, S. Raymond, H. Feilbach, C. Mounier, B. Vettard, and T. Brückel, The upgrade of the cold neutron three-axis spectrometer IN12 at the ILL, Nucl. Instruments Methods A 819, 89 (2016).

[61] The neutron scattering data recorded on the IN12 spectrometer at the Institut Laue-Langevin (ILL) are available from http://dx. doi.org/10.5291/ILL-DATA.4-01-1327 (2013).

[62] M. Boehm, P. Steffens, J. Kulda, M. Klicpera, S. Roux, P. Courtois, P. Svoboda, J. Saroun, and V. Sechovsky, ThALES- 
Three axis low energy spectroscopy for highly correlated electron systems, Neutron News 26, 18 (2015).

[63] The neutron scattering data recorded on the ThALES spectrometer at the Institut Laue-Langevin (ILL) are available from https://doi.ill.fr/10.5291/ILL-DATA.TEST-2473 (2015).

[64] The neutron scattering data recorded on the ThALES spectrometer at the Institut Laue-Langevin (ILL) are available from http://dx.doi.org/10.5291/ILL-DATA.4-02-563 (2020).

[65] H. Jacobsen, S. L. Holm, M. E. Lăcătuşu, A. T. Rømer, M. Bertelsen, M. Boehm, R. Toft-Petersen, J. C. Grivel, S. B. Emery, L. Udby, B. O. Wells, and K. Lefmann, Distinct Nature of Static and Dynamic Magnetic Stripes in Cuprate Superconductors, Phys. Rev. Lett. 120, 037003 (2018).

[66] A. T. Rømer, P. J. Ray, H. Jacobsen, L. Udby, B. M. Andersen, M. Bertelsen, S. L. Holm, N. B. Christensen, R. Toft-Petersen, M. Skoulatos, M. Laver, A. Schneidewind, P. Link, M. Oda, M. Ido, N. Momono, and K. Lefmann, Field-induced interplanar magnetic correlations in the high-temperature superconductor $\mathrm{La}_{1.88} \mathrm{Sr}_{0.12} \mathrm{CuO}_{4}$, Phys. Rev. B 91, 174507 (2015).

[67] F. Tasset, Zero field neutron polarimetry, Phys. B: Condens. Matter 156, 627 (1989).

[68] B. O. Wells, R. J. Birgeneau, F. C. Chou, Y. Endoh, D. C. Johnston, M. A. Kastner, Y. S. Lee, G. Shirane, J. M. Tranquada, and K. Yamada, Intercalation and staging behavior in super-oxygenated $\mathrm{La}_{2} \mathrm{CuO}_{4+\delta}$, Z. Phys. B 100, 535 (1996).

[69] Y. S. Lee, F. C. Chou, A. Tewary, M. A. Kastner, S. H. Lee, and R. J. Birgeneau, Neutron scattering study of the effects of dopant disorder on the superconductivity and magnetic order in stage-4 $\mathrm{La}_{2} \mathrm{CuO}_{4+y}$, Phys. Rev. B 69, 020502(R) (2004).

[70] G. Shirane, S. M. Shapiro, and J. M. Tranquada, Neutron Scattering with a Triple-Axis Spectrometer (Cambridge University Press, Cambridge, UK, 2002).

[71] G. Xu, Z. Xu, and J. M. Tranquada, Absolute cross-section normalization of magnetic neutron scattering data, Rev. Sci. Instrum. 84, 083906 (2013).

[72] H. Sato and K. Maki, Theory of inelastic neutron scattering from $\mathrm{Cr}$ and its alloys near the Neel temperature, Int. J. Magn. 6, 183 (1974).

[73] G. Aeppli, T. E. Mason, S. M. Hayden, H. A. Mook, and J. Kulda, Nearly singular magnetic fluctuations in the normal state of a high- $\mathrm{T}_{c}$ cuprate superconductor, Science 278, 1432 (1997).

[74] Y. Ando, G. S. Boebinger, A. Passner, L. F. Schneemeyer, T. Kimura, M. Okuya, S. Watauchi, J. Shimoyama, K. Kishio, K. Tamasaku et al., Resistive upper critical fields and irreversibility lines of optimally doped high- $\mathrm{T}_{c}$ cuprates, Phys. Rev. B 60, 12475 (1999).

[75] H. Jacobsen, S. M. Gaw, A. J. Princep, E. Hamilton, S. Tóth, R. A. Ewings, M. Enderle, E. M. Hetroy Wheeler, D.
Prabhakaran, and A. T. Boothroyd, Spin dynamics and exchange interactions in $\mathrm{CuO}$ measured by neutron scattering, Phys. Rev. B 97, 144401 (2018).

[76] A. Boothroyd, Principles of Neutron Scattering from Condensed Matter (Oxford University Press, Oxford, 2020).

[77] M. Braden, G. Heger, P. Schweiss, Z. Fisk, K. Gamayunov, I. Tanaka, and $\mathrm{H}$. Kojima, Characterization and structural analysis of twinned $\mathrm{La}_{2-x} \mathrm{Sr}_{x} \mathrm{CuO}_{4 \pm \delta}$ crystals by neutron diffraction, Physica C 191, 455 (1992).

[78] T. Imai and Y. S. Lee, ${ }^{139} \mathrm{La}$ and ${ }^{63} \mathrm{Cu}$ NMR investigation of charge order in $\mathrm{La}_{2} \mathrm{CuO}_{4+y}\left(T_{c}=42 \mathrm{~K}\right)$, Phys. Rev. B 97, 104506 (2018).

[79] L. Udby, J. Larsen, N. B. Christensen, M. Boehm, C. Niedermayer, H. E. Mohottala, T. B. S. Jensen, R. ToftPetersen, F. C. Chou, N. H. Andersen, K. Lefmann, and B. O. Wells, Measurement of Unique Magnetic and Superconducting Phases in Oxygen-Doped High-Temperature Superconductors $\mathrm{La}_{2-x} \mathrm{Sr}_{x} \mathrm{CuO}_{4+y}$, Phys. Rev. Lett. 111, 227001 (2013).

[80] M. H. Christensen, H. Jacobsen, T. A. Maier, and B. M. Andersen, Magnetic Fluctuations in Pair-Density-Wave Superconductors, Phys. Rev. Lett. 116, 167001 (2016).

[81] B. Vignolle, S. M. Hayden, D. F. McMorrow, H. M. Rønnow, B. Lake, C. D. Frost, and T. G. Perring, Two energy scales in the spin excitations of the high-temperature superconductor $\mathrm{La}_{2-x} \mathrm{Sr}_{x} \mathrm{CuO}_{4}$, Nat. Phys. 3, 163 (2007).

[82] J. Chang, C. Niedermayer, R. Gilardi, N. B. Christensen, H. M. Rønnow, D. F. McMorrow, M. Ay, J. Stahn, O. Sobolev, A. Hiess, S. Pailhes, C. Baines, N. Momono, M. Oda, M. Ido, and J. Mesot, Tuning competing orders in $\mathrm{La}_{2-x} \mathrm{Sr}_{x} \mathrm{CuO}_{4}$ cuprate superconductors by the application of an external magnetic field, Phys. Rev. B 78, 104525 (2008).

[83] P. G. Radaelli, J. D. Jorgensen, A. J. Schultz, B. A. Hunter, J. L. Wagner, F. C. Chou, and D. C. Johnston, Structure of the superconducting $\mathrm{La}_{2} \mathrm{CuO}_{4+\delta}$ phases $(\delta \simeq 0.08,0.12)$ prepared by electrochemical oxidation, Phys. Rev. B 48, 499 (1993).

[84] A.-E. Ţuţueanu, M. Sales, K. L. Eliasen, M.-E. Lăcătuşu, J.-C. Grivel, N. Kardjilov, I. Manke, M. Krzyzagorski, Y. Sassa, M. S. Andersson, S. Schmidt, and K. Lefmann, Non-destructive characterisation of dopant spatial distribution in cuprate superconductors, Physica C 575, 1353691 (2020).

[85] J. E. Sonier, J. H. Brewer, and R. F. Kiefl, $\mu$ SR studies of the vortex state in type-II superconductors, Rev. Mod. Phys. 72, 769 (2000).

[86] K. Momma and F. Izumi, VESTA: A three-dimensional visualization system for electronic and structural analysis, J. Appl. Crystallogr. 41, 653 (2008).

[87] P. J. Brown, Form factors for $3 d$ transition elements and their ions, https://www.ill.eu/sites/ccsl/ffacts/ffactnode5.html. 